\title{
D. CERVEAU
}

R. MOUSSU

\section{Groupes d'automorphismes de $(\mathbb{C}, 0)$ et équations} différentielles $y d y+\cdots=0$

Bulletin de la S. M. F., tome 116, no 4 (1988), p. 459-488

<http://www.numdam.org/item?id=BSMF_1988_116_4_459_0>

(C) Bulletin de la S. M. F., 1988, tous droits réservés.

L'accès aux archives de la revue «Bulletin de la S. M. F. » (http: //smf.emath.fr/Publications/Bulletin/Presentation.html) implique l'accord avec les conditions générales d'utilisation (http://www.numdam.org/ conditions). Toute utilisation commerciale ou impression systématique est constitutive d'une infraction pénale. Toute copie ou impression de ce fichier doit contenir la présente mention de copyright.

\section{Numdam}


Bull. Soc. math. France,

116, 1988, p. 459-488.

\title{
GROUPES D'AUTOMORPHISMES DE (C,0) ET ÉQUATIONS DIFFÉRENTIELLES $y d y+\cdots=0$
}

PAR

\author{
D. CERVEAU ET R. MOUSSU
}

RÉSumÉ. - Dans cet article, on étudie la classification analytique des groupes non abéliens de germes de difféomorphismes de $\mathbb{C}, 0$. On en déduit la classification de certains diagrammes divergents et d'équations différentielles singulières dans le plan complexe.

ABSTRACT. - We study the analytic classification of non abelian groupes of diffeomorphisms of $\mathbb{C}, 0$. We apply our results to the classification of divergent diagrams and to singular ordinary differential equations in $\mathbb{C}^{2}$. Space of moduli are given.

\section{Introduction}

Soit $\mathbb{D}$ le groupe des germes d'automorphismes holomorphes de $\mathbb{C}$ au point 0. L'étude des sous-groupes de $\mathbb{D}$ est étroitement liée via la monodromie à celles des singularités des équations différentielles holomorphes du plan complexe.

Les problèmes rencontrés sont de nature différente selon que l'objet étudié est formellement linéarisable ou ne l'est pas. Dans le premier cas, on tombe dans le monde des petits diviseurs $[\mathrm{H}]$, dans le second on est dans celui de la resommabilité, des fonctions résurgentes $\left[E_{1}\right],\left[E_{2}\right],\left[M, R_{1}\right]$, $\left[\mathrm{M}, \mathrm{R}_{2}\right]$.

Le but de ce travail est de classifier les sous-groupes non abéliens de D et ensuite d'en déduire des propriétés des équations différentielles du type $y d y+\cdots=0$. De tels groupes possèdent des éléments tangents à l'application identique $1_{\mathbb{C}}$ de $\mathbb{C}$. Ils ne sont pas linéarisables et la resommabilité va jouer un rôle fondamental; c'est une des clefs de la preuve du THÉORÈME 1.

$\left.{ }^{*}\right)$ Texte reçu le 11 juin 1987 .

D. Cerveau, IRmar, Université de Rennes I, 35042 Rennes Cedex, France.

R. Moussu, Université de Bourgogne, Laboratoire de Topologie, B.P. 138, 21004 Dijon Cedex, France. 
Avant de l'énoncer, rappelons quelques notations et définitions classiques. On note $\widehat{\mathbb{D}}$ le complété formel de $\mathbb{D}$ et $\Phi_{*}$ l'automorphisme intérieur de $\widehat{\mathbb{D}}, g \rightarrow \Phi \circ g \circ \Phi^{-1}$ correspondant à l'élément $\Phi$ de $\widehat{\mathbb{D}}$. Deux sous-groupes $G, G^{\prime}$ de $\mathbb{D}$ sont holomorphiquement (resp. formellement) conjugués s'il existe un élément $\Phi$ de $\mathbb{D}$ (resp. $\widehat{\mathbb{D}})$ tel que $\Phi_{*} G=G^{\prime}$.

Un sous-groupe $G$ de $\mathbb{D}$ est dit exceptionnel s'il est formellement conjugué à l'un des groupes $G_{\omega, p}, p \in N^{*}, \omega \in \mathbb{C}$, engendré par

$$
x \rightarrow \omega x \quad \text { et } \quad h_{p}(x)=x\left(1-p x^{p}\right)^{-1 / p} \quad \text { avec } \quad \omega^{p}=-1,(1)^{1 / p}=1 .
$$

ThÉORÈme 1. - Deux sous-groupes non abéliens $G, G^{\prime}$ de $\mathbb{D}$ formellement conjugués le sont holomorphiquement si $G$ n'est pas exceptionnel.

Nous montrerons que, lorsque $G$ est exceptionnel, son espace de module (formel-holomorphe) s'identifie à un sous-ensemble de l'espace des modules d'ECALle-Voronin de l'élément $h_{p}$ de $\mathbb{D}$.

Dans le chapitre 2, nous appliquerons le THÉORÈme 1 aux diagrammes $d$ à deux flèches $\left(f_{1}, f_{2}\right) \in \mathbb{C}\{x\}^{2}$ via son groupe d'invariance $G(d)$. Le diagramme $d$ est dit exceptionnel si $G(d)$ est exceptionnel. Par exemple un diagramme dont une des flèches est d'ordre impair n'est jamais exceptionnel.

ThÉoRÈme 2. - Deux diagrammes $d, d^{\prime}$ formellement conjugués le sont holomorphiquement si d n'est pas exceptionnel.

Il est bien connu [T] qu'une équation différentielle $\Omega=0$ de 1 -jet $y d y$ a une "forme normale" du type

$$
\Omega_{n, p}=d\left(y^{2}+x^{n}\right)+x^{p} U(x) d y, \quad U(x) \in \mathbb{C}[[x]], \quad U(0) \neq 0 .
$$

L'entier $n$ est un invariant formel de $\Omega$ et nous notons $\Sigma_{1}^{n}$ l'ensemble des $\Omega$ qui ont une forme normale $\Omega_{n, p}$ avec $2 p>n$. Une telle équation "hérite" de nombreuses propriétés de l'hamiltonien $y^{2}+x^{n}$. En particulier, elle a la même résolution minimale $M$ que la courbe $y^{2}+x^{n}=0$. En remarquant que le groupe d'holonomie $G_{\Omega}$ d'un diviseur de $M$ caractérise $\Omega$ on montre le :

ThÉORÈme 3. - Deux équations $\Omega, \Omega^{\prime} \in \Sigma_{1}^{n}$ formellement conjuguées le sont holomorphiquement sauf peut-être dans le cas exceptionnel : $n$ est pair, $G_{\Omega}$ est abélien et $\Omega$ n'a pas d'intégrale première holomorphe.

Le chapitre 4 est consacré à la classification des sous-groupes de $\mathbb{D}$ topologiquement conjugués à des sous-groupes non abéliens du groupe $\mathbf{H}$ constitué des germes d'homographies

$$
x \rightarrow \frac{a x}{1+b x} \quad \text { où } \quad a, b \in \mathbb{C} .
$$

TOME $116-1988-\mathrm{N}^{\circ} 4$ 
De l'étude des représentations fidèles des sous-groupes de $H$ dans $\mathbb{D}$ on déduit le :

Corollaire 1. - Un sous-groupe $G$ de $\mathbb{D}$ topologiquement conjugué à un sous-groupe non abélien $H$ de $\mathrm{H}$ est formellement conjugué à un sousgroupe de $\mathbb{H}$. De plus, cette conjugaison est holomorphe si $H$ est différent $d u$ groupe exceptionnel $G_{-1,1}=\{-x,(x / 1-x)\}$.

Soit $H$ un sous-groupe de $\mathbb{H}$, tel que $J^{1} H$ soit réel; si $L$ est un élément de $\mathrm{GL}(2, \mathbb{R})$ on introduit le groupe $L_{*} H$ :

$$
L_{*} H=\left\{x \rightarrow \frac{a x}{1+L(b) x} ; \frac{a x}{1+b x} \in H\right\} .
$$

Théorème 4. - Soit $H \neq G_{-1,1}$ un sous-groupe non abélien de $\mathbf{H}$ et soit $G$ un sous-groupe de $\mathbb{D}$ topologiquement conjugué à $H$. Alors si :

i) $J^{1} H$ n'est pas réel, $G$ est holomorphiquement conjugué à $H$.

ii) $J^{1} H$ est réel, $G$ est holomorphiquement conjugué à un sous-groupe $L_{*}(H)$ avec $L \in \mathrm{GL}(2, \mathbb{R})$.

\section{Notations}

$$
\begin{aligned}
\mathbb{D} & =\left\{h \in \mathbb{C}\{x\}, h(0)=0, h^{\prime}(0) \neq 0\right\} ; \\
\mathbb{D}_{p} & =\left\{h \in \mathbb{D}, h^{(i)}(0)=0,2 \leq i \leq p, h^{(1)}(0)=1\right\} ;
\end{aligned}
$$

$\widehat{\mathbb{D}}, \widehat{\mathbb{D}}_{p}$ les complétés formels de $\mathbb{D}$ et $\mathbb{D}_{p}$;

$$
\begin{aligned}
& X_{p, \lambda}=\frac{x^{p}}{1+\lambda x^{p+1}} \cdot \frac{\partial}{\partial x} \\
& G_{\omega, p} \text { le groupe engendré par } x \rightarrow \omega x, \omega^{p}=-1, \text { et } \exp X_{p, 0} .
\end{aligned}
$$

Pour $\Phi \in \widehat{\mathbb{D}}$ ou $\mathbb{D}$ on note $\Phi_{*}$ l'automorphisme de $\widehat{\mathbb{D}}$ ou $\mathbb{D}: g \mapsto \Phi \circ g \circ \Phi^{-1}$. Si $h \in \mathbb{D}$, on note $[h]_{\mathbb{D}}$ (resp. $[h]_{\widehat{\mathbb{D}}}$ ) l'ensemble $\left\{\Phi_{*} h, \Phi \in \mathbb{D}\right\}$ (respectivement $\left.\left\{\Phi_{*} h, \Phi \in \widehat{\mathbb{D}}\right\} \cap \mathbb{D}\right)$.

De même, si $G$ est un sous-groupe de $\mathbb{D},[G]_{\mathbf{D}}$ désigne $\left\{\Phi_{*} G, \Phi \in \mathbb{D}\right\}$; l'espace des modules $G^{\text {For }}$ de $G$ est l'ensemble des $\left[G^{\prime}\right]_{\text {D }}$ où $G^{\prime}$ est formellement conjugué à $G$.

Remerciements. - C'est après une discussion avec Jean Martinet que nous avons vraiment démarré cette étude. Nous le remercions vivement. 
0. Rappels sur les automorphismes de $(\mathbb{C}, 0)$ tangents à $1_{\mathbb{C}}$

1. Classification formelle. - Elle se déduit de la classification des champs de vecteurs à une variable. Soit

$$
X=x^{p+1} f(x) \frac{\partial}{\partial x}, \quad f \in \mathbb{C}[[x]], \quad f(0) \neq 0
$$

un champ de vecteurs formel qui s'annule à l'ordre $p$ en 0 et soit $\exp t X$, $t \in \mathbb{C}$, son groupe à 1 -paramètre, i.e. :

$$
\frac{d}{d t} \exp t X=X(\exp t X) \quad \text { avec } \quad \exp 0 X=1_{\mathrm{C}} .
$$

En développant cette relation suivant les puissances de $x$, on montre que $\exp t X(x)$ est un élément de $\mathbb{C}[t][[x]]$. On en déduit l'assertion :

1.1. - $X$ est déterminé par $\exp X$ en ce sens : si un autre champ $X^{\prime}$ est relié à $X$ par :

$$
g_{*} \exp X=\exp X^{\prime} \quad \text { où } \quad g \in \widehat{\mathbb{D}}
$$

alors $g_{*} X=X^{\prime}$.

En considérant la 1-forme $\frac{d x}{x^{p+1} f(x)}$ duale de $X$, on établit :

1.2. - Il existe $p \in \mathbb{N}, \lambda \in \mathbb{C}$ unique et $N \in \widehat{\mathbb{D}}$ tels que :

$$
N_{*} X=X_{p, \lambda} \quad \text { où } X_{p, \lambda}=\frac{x^{p+1}}{1+\lambda x^{p}} \frac{\partial}{\partial x} .
$$

L'assertion suivante caractérise les éléments $g \in \widehat{\mathbb{D}}$ qui laissent $X_{p, \lambda}$ fixe, ou ce qui revient au même, qui commutent avec $\exp X_{p, \lambda}$. Elle se démontre en résolvant $E_{1}$ où $E_{\mu}$ désigne l'équation différentielle :

$$
\frac{g^{\prime}}{g^{p+1}}+\frac{\lambda g^{\prime}}{g}=\mu\left(\frac{1}{x^{p+1}}+\frac{\lambda}{x}\right), \quad \mu \in \mathbb{C} .
$$

1.3. - Si $g_{*} X_{p, \lambda}=X_{p, \lambda}$ avec $g \in \widehat{\mathbb{D}}$, il existe $t_{g} \in \mathbb{C}$ et $k \in$ $\{0,1, \ldots, p-1\}$ tels que :

$$
g(x)=e^{2 i \pi(k / p)} \exp t_{g} X_{p, \lambda} .
$$

TOME $116-1988-\mathrm{N}^{\circ} 4$ 
L'Assertion 1.4. est un critère de nullité de l'invariant $\lambda$ de $X_{p, \lambda}$. Elle se prouve comme 1.3. en intégrant $E_{\mu}$.

1.4. - Si $g_{*} X_{p, \lambda}=\mu X_{p, \lambda}$ avec $\mu \neq 1$ et $g \in \widehat{\mathbb{D}}$ alors $\lambda=0$ et

$$
g(x)=a \exp t_{g} X_{p, 0} \quad \text { où } \quad t_{g} \in \mathbb{C}
$$

et $a \in \mathbb{C}$ vérifie $\mu a^{p}=1$. L'application $X \rightarrow \exp X$ est surjective; plus précisément on a :

1.5. - Soit $h(x)=x\left(1+\sum_{k \geq p} a_{k} x^{k}\right), a_{p} \neq 0$, un élément de $\widehat{\mathbb{D}}_{1}$. Il existe $\lambda \in \mathbb{C}$ unique et $N \in \widehat{\mathbb{D}}$ tangent à $1_{\mathbb{C}}$ tels que :

$$
N_{*} h=\exp a_{p} X_{p, \lambda} .
$$

$N$ est appelée une transformation normalisante de $h$ (itérateur chez [E]). De 1.3., 1.4., 1.5., on déduit :

1.6. - Le centralisateur $\widehat{C}(h)=\left\{g \in \widehat{\mathbb{D}} ; g_{*} h=h\right\}$ de $h$ dans $\widehat{\mathbb{D}}$ est le groupe engendré par les

$$
N_{*}^{-1}\left(e^{2 i \pi(k / p)} \exp t X_{p, \lambda}\right) \quad \text { avec } t \in \mathbb{C}, \quad k=0,1, \ldots, p-1 .
$$

En particulier $\widehat{C}_{1}(h)=\widehat{C}(h) \cap \widehat{\mathbb{D}}_{1}$ est le groupe à 1-paramètre

$$
N_{*}^{-1} \exp t X_{p, \lambda}
$$

et ainsi les composés $\exp t X_{p, \lambda} \circ N$ sont les seules transformations normalisantes de $h$. On en déduit que $h$ possède une unique transformation normalisante tangente à $1_{\mathbb{C}}$ à l'ordre $p$.

2. Classification topologique. - Elle est due à C. СамаCHo [C].

2.1. - Les éléments de $\mathbb{D}_{p}-\mathbb{D}_{p+1}$ sont tous topologiquement conjugués. Il est naturel de prendre pour représentant de cette classe de conjugaison

$$
h_{p}(x)=\exp X_{p, 0}(x)=\frac{x}{\sqrt[p]{1-p x^{p}}} \quad \text { avec } \quad \sqrt[p]{1}=1 .
$$

A une homothétie près $h_{p}(x)$ est un relèvement de $h_{1}(x)$ par le revêtement ramifié $x \rightarrow x^{p}$. Sa dynamique (théorème de la fleur) se visualise de la façon suivante : 
2.2. - Les $2 p$ droites $d_{\ell}=\left\{x ; \operatorname{Arg} x=\ell(\pi / p), \ell=0,1, \ldots, 2_{p-1}\right\}$ sont invariantes par $h_{p}$; nous les appelons rayons invariants. Si $x$ appartient à l'un des secteurs

$$
S_{\ell}=\left\{x \in \mathbb{C} / \ell \cdot \frac{\pi}{p}<\operatorname{Arg} x<(\ell+1) \frac{\pi}{p}\right\}
$$

$h_{p}^{n}(x)$ tend vers 0 sur une courbe analytique tangente à $d_{\ell}$ ou $d_{\ell+1}$ lorsque $n$ tend vers $\pm \infty$.

Cette dynamique est préservée par conjugaison topologique, plus précisément :

2.3. - Soient $h(x)=x\left(1+\sum_{k \geq p} a_{k} x^{k}\right)$ un élément de $\mathbb{D}_{p}-\mathbb{D}_{p+1}$, $d_{\ell}(h)$ la demi-droite image de $d_{\ell}$ par la rotation d'angle $1 / p \operatorname{Arg} a_{p}$. Si $x$ est voisin de 0 , une au moins des demi orbites $\left\{h^{\epsilon \cdot n}(x)\right\}_{n \in \mathbf{N}}, \epsilon= \pm 1$, tend vers zéro le long d'une courbe (topologique) tangente en 0 à une des demi-droites $d_{\ell}(h)$. Nous dirons encore que les $d_{\ell}(h)$ sont des rayons invariants.

3. Classification analytique. - Les résultats ci-dessous ont été obtenus tout d'abord par J. ECALLE [E], (via la théorie des fonctions résurgentes) puis par M. Voronin (via des conjugaisons quasi-conformes) [V]. Ils ont été exposés par B. Malgrange dans $[\mathrm{M}]$ et reformulés (via la resommabilité) par J. MARTINET et J.-P. RAMIS dans [MR $\left.{ }_{1}\right]$ et $\left[\mathrm{MR}_{2}\right]$.

Soit $N$ une transformation normalisante d'un élément $h(x)=x(1+$ $\left.\sum_{k \geq p} a_{k} x^{k}\right), a_{p} \neq 0$, i.e. $N_{*} h=\exp a_{p} X_{p, \lambda}$ et soit $S_{\ell}^{\prime}(h)$ le secteur d'ouverture $2 \pi / p$ bissecté par $d_{\ell}(h)$. L'assertion suivante est connue sous le nom de :

3.1. Normalisation sectorielle. - il existe un germe unique $N_{\ell}$ d'automorphisme holomorphe de $\left(S_{\ell}^{\prime}(h), 0\right)$ dont $N$ est le développement asymptotique en 0 et tel que :

$$
N_{\ell^{*}} h=\exp a_{p} X_{p, \lambda} \mid\left(S^{\prime}(h), 0\right) .
$$

3.2. Cocycles caractéristiques. - C'est la collection $c(h)$ des germes d'automorphismes

$$
c_{\ell}(h)=N_{\ell+1} \circ N_{\ell}^{-1}:\left(S_{\ell+1}^{\prime}(h) \cap S_{\ell}(h), 0\right) \circlearrowleft ;
$$

$c(h)$ caractérise la classe de conjugaison holomorphe $[h]_{\mathbf{D}}:$ si $h_{1}$ appartient $\grave{\mathrm{a}}[h]_{\widehat{\mathrm{D}}}$, alors $h_{1} \in[h]_{\mathbf{D}}$ si et seulement si $c(h)=c\left(h_{1}\right)$. Lorsque $N$ converge $c(h)$ est évidemment trivial et le centralisateur holomorphe $C_{1}(h)$ coïncide

TOME $116-1988-\mathrm{N}^{\circ} 4$ 
avec le centralisateur formel $\widehat{C}_{1}(h)=\left\{g \in \widehat{\mathbb{D}} ; g_{*} h=h\right\}$. Plus précisément on a :

3.3. - $\quad N$ diverge si et seulement si $C_{1}(h)$ est monogène ([E], [V]).

\section{Espaces des modules formels des sous-groupes de $\mathbb{D}$}

1. Énoncé des résultats. - Soit $G$ un sous-groupe de D. Son espace de modules (formels-holomorphes), noté $G^{\text {For }}$, est l'ensemble des $\left[G^{\prime}\right]_{\mathrm{D}}$ où $G^{\prime}$ est un sous-groupe de $\mathbb{D}$ formellement conjugué à $G$ et $\left[G^{\prime}\right]_{\mathbb{D}}$ est l'ensemble des sous-groupes de $\mathbb{D}$ holomorphiquement conjugués à $G^{\prime}$.

D'après l'assertion 0.3.2., lorsque $G \subset \mathbb{D}_{1}$ est un groupe monogène (soit $\left.\left\{h^{n} ; n \in \mathbb{Z}\right\}\right), G^{\text {For }}$ n'est rien d'autre que le module d'ECALLE-Voronin, $h^{\text {For }}$ de $h$ qui s'identifie à l'ensemble des cocycles $c\left(h^{\prime}\right)$ pour $h^{\prime} \in[h]_{\widehat{\mathbf{D}}} \cap \mathbb{D}$.

Lorsque $G$ est un sous-groupe abélien de $\mathbb{D}$, la détermination $\operatorname{de} G^{\text {For }}$ est souvent liée à des problèmes de petits diviseurs; c'est donc un problème très difficile; nous ne l'étudierons pas ici. Par contre, si $G$ n'est pas abélien, le théorème suivant montre que $G^{\text {For }}$ est trivial si $G$ n'est pas un des groupes exceptionnels $G_{\omega, p}$ engendrés par $\exp X_{p, 0}$ et $x \mapsto \omega x$ avec $\omega^{p}=-1$.

THÉORÈme 1. - Soit $G$ un sous-groupe non abélien de $\mathbb{D}$.

i) Si $G^{\prime} \subset \mathbb{D}$ est formellement conjugué à $G$ par $\Phi \in \widehat{\mathbb{D}}$, alors, $\Phi$ converge dès que $G$ n'est pas formellement conjugué à l'un des groupes $G_{\omega, p}$

ii) L'espace $G_{\omega, p}^{\mathrm{For}} s^{\prime}$ 'identifie au sous-ensemble de $\left(\exp X_{p, 0}\right)^{\text {For }}$ constitué des cocycles $\left(c=c_{0}, c_{1}, \ldots, c_{2 p-1}\right)$ qui sont équivariants par la rotation $x \rightarrow \omega x ; c^{\prime} e s t-\grave{a}$-dire :

$$
c_{\ell+d}(\omega x)=\omega c_{\ell}(x) \quad \text { si } \omega=e^{i \pi(d / p)}, \omega^{p}=-1 .
$$

Dans [V], Voronin classifie les couples $\left(f_{1}, f_{2}\right)$ d'involutions génériques. En remarquant (voir Proposition 2 ci-dessous) que le groupe engendré par $f_{1}, f_{2}$ est alors formellement conjugué à $G_{-1,1}=\{-x,(x / 1-x)\}$, nous retrouvons son résultat : l'espace des modules d'une paire d'involutions s'identifie à l'ensemble des cocycles de $(x / 1-x)^{\text {For }}$ invariants par l'involution $x \mapsto-x$.

La motivation première de ce travail était le problème plus général lié aux équations différentielles : classifier les paires $\left(f_{1}, f_{2}\right)$ où les $f_{i} \in \mathbb{D}$ sont périodiques. Le ThÉorème 1 résout ce problème compte tenu de la remarque :

Remarque 1. - Un système de générateurs $\left(f_{1}, f_{2}\right)$ périodiques du 
groupe $G_{\omega, p}, \omega^{p}=-1$ est à conjugaison holomorphe près du type suivant :

$$
f_{1}(x)=e^{i \pi \cdot\left(n_{1} / p\right)} \cdot x, \quad f_{2}(x)=e^{i \pi \cdot\left(n_{2} / p\right)} \frac{x}{\sqrt[p]{1-x^{p}}},
$$

avec $n_{1}$ et $n_{2}$ impairs.

En effet, ces deux éléments engendrent un sous-groupe $G_{n_{1}, n_{2}, d}$ de $\mathbf{D}$ non abélien tel que $G_{n_{1}, n_{2}, d} \cap \mathbb{D}_{1}$ soit monogène; $G_{n_{1}, n_{2}, p}$ est holomorphiquement conjugué à un groupe $G_{\omega, p}$ d'après la Proposition 2 ci-dessous. Réciproquement toute paire d'éléments $f_{1}, f_{2}$ de $G_{\omega, p}$ s'écrit :

$$
f_{j}(x)=\omega^{m_{j}} \frac{x}{\sqrt[p]{1-k_{j} x^{p}}}, \quad m \in \mathbb{N}, k_{j} \in \mathbb{Z} .
$$

On vérifie que, si les $f_{j}$ sont périodiques et ne commutent pas, les entiers $m_{j}$ sont impairs. Ainsi,

$$
\omega^{m_{j}}=e^{i(\pi / p) \cdot n_{j}} \quad \text { avec } \quad n_{j}=m_{j}(2 d+1) \quad \text { si } \quad \omega=e^{i \pi(2 d+1 / p)}
$$

et $\left(f_{1}, f_{2}\right)$ est du type annoncé.

Le corollaire suivant est alors une conséquence du théorème et des propriétés des $G_{\omega, p}$.

Corollaire 1. - Soient $G, G^{\prime}$ deux sous-groupes non abéliens de D formellement conjugués par $\Phi \in \widehat{\mathbb{D}}$. Alors $\Phi$ converge dans les cas suivants :

1) G n'est pas engendré par deux éléments.

2) $G$ ne contient pas d'élément périodique.

3) $G$ est engendré par deux éléments périodiques dont l'un est de période impaire.

2. Démontration du théorème. - C'est une conséquence des Propositions 1, 2 suivantes.

Proposition 1. - Soient $G, G^{\prime}$ deux sous-groupes de $\mathbf{D}_{1}$ formellement conjugués par $\Phi \in \widehat{\mathbb{D}}$. Si $G$ n'est pas monogène, $\Phi$ converge.

Preuve. - Modulo une conjugaison linéaire, on peut supposer que $\Phi$ appartient à $\widehat{\mathbf{D}}_{1}$. Distinguons deux cas :

1) $G$ est abélien non monogène. Soit $h$ un élément (fixé) de $G$. Comme $G$ est inclus dans le centralisateur $C_{1}(h)$, les transformations normalisantes de $h$ sont convergentes; soit $N$ l'une d'entre elles. Le composé $N^{\prime}=\Phi^{-1} \circ N$ est une transformation normalisante de $h^{\prime}=\Phi_{*} h$. Elle converge par le même argument que précédemment et par suite $\Phi$ converge.

$$
\text { TOME } 116-1988-\mathrm{N}^{\circ} 4
$$


2) $G$ n'est pas abélien. Soient $h_{i}, i=1,2$ deux éléments de $G$ non commutants :

$$
h_{i}(x)=x\left(1+t_{i} x^{p_{i}}+\cdots\right), \quad t_{i} \neq 0 .
$$

Lorsque $p_{1}=p_{2}$, un calcul immédiat montre que $\left[h_{1}, h_{2}\right]$ est tangent à l'identité à un ordre strictement supérieur à $p_{1}$. On peut donc choisir deux éléments $h_{1}, h_{2} \in G$ tels que $p_{1} \neq p_{2}$. La transformation $\Phi$ conjugue les difféomorphismes holomorphes $h_{i}$ et $\Phi_{*} h_{i}$ pour $i=1,2$. Un argument de [MR $\left.\mathrm{MR}_{1}\right]$ assure qu'elle est $p_{i}$-sommable pour $i=1,2$ et d'après un résultat de J.-P. RAmis [R] elle est convergente.

La proposition suivante classifie les sous-groupes $G$ de $\widehat{\mathbb{D}}$ tels que $G \cap \widehat{\mathbb{D}}_{1}$ soit monogène.

Proposition 2. - Soit $G$ un sous-groupe de $\mathbb{D}$ tel que $G \cap \mathbb{D}$ soit engendré par $h(x)=x\left(1+x^{p}+\cdots\right)$ et soit $N$ une transformation normalisante de $h, N_{*} h=\exp X_{p, \lambda}$. Il existe $f \in G$ tel que $f$ et $h$ engendrent $G, f_{*} h=h^{\epsilon}$ avec $\epsilon= \pm 1$ et pour un bon choix de $N$ on a :

i) si $\epsilon=1, G$ est abélien et $N_{*} G$ est engendré par $\exp X_{p, \lambda}$ et $\omega \exp t \cdot X_{p, \lambda}$ où $t \in \mathbb{C}$ et $\omega^{p}=1$.

ii) si $\epsilon=-1$, alors $\lambda=0$ et $N_{*} G$ est un des groupes $G_{\omega, p}$ (avec $\left.\omega^{p}=-1\right)$.

Preuve. - Si $g$ est un élément de $G, g_{*} h$ appartient à $G \cap \mathbb{D}_{1}$ et il existe $n(g) \in \mathbb{Z}$ tel que :

$$
g_{*} h=h^{n(g)}=x\left(1+n(g) x^{p}+\cdots\right) .
$$

D'autre part un calcul élémentaire montre que

$$
g_{*} h=x\left(1+g^{\prime}(0)^{-p} x^{p}+\cdots\right) .
$$

En procédant de la même façon avec $g^{-1}$ on obtient :

$$
n(g)=g^{\prime}(0)^{-p}, \quad g^{\prime}(0)^{p}=n\left(g^{-1}\right), \quad n(g)=\epsilon \quad \text { avec } \quad \epsilon= \pm 1
$$

Le sous-groupe $J^{1} G=\left\{g^{\prime}(0) ; g \in G\right\}$ est un sous-groupe des racines $2^{p \text { ième }}$ de 1 . Soient $\omega$ un générateur de $J^{1} G$ et $f \in G$ tel que $f^{\prime}(0)=\omega$; quel que soit $g \in G$, il existe $m(g) \in \mathbb{Z}$ tel que $g^{\prime}(0)=\omega^{m(g)}$. Ainsi $f^{-m(g)} \cdot g$ appartient à $G \cap \mathbb{D}_{1}$, c'est-à-dire à $\left\{h^{n}\right\}_{n \in \mathbf{Z}}$. Ce qui démontre la première partie de la proposition : $G$ est engendré par $f, h$ avec

$$
f_{*} h=h^{\epsilon}, \quad f^{\prime}(0)=\omega, \quad \omega^{p}=\epsilon= \pm 1 .
$$

Soit $N$ une transformation normalisante de $h, N_{*} h=\exp X_{p, \lambda}$ : 
i) si $\epsilon=1, G$ est abélien et d'après l'assertion 0.1.6

$$
N_{*} f=\omega \exp t X_{p, \lambda} \quad \text { avec } t \in \mathbb{C} .
$$

ii) si $\epsilon=-1$, d'après 0.1 .4 . on a $\lambda=0$ et $N_{*} f=\omega \exp t X_{p, 0}$ puisque :

$$
\left(N_{*} f\right)_{*} \exp X_{p, 0}=\exp -X_{p, 0} .
$$

On peut ici choisir $N$ tel que $N_{*} f(x)=\omega x$.

\section{Diagrammes divergents}

Définition. - On appelle diagramme (à deux flèches) une paire $d=\left(f_{1}, f_{2}\right)$ d'éléments $f_{j}$ de $\mathbb{C}\{x\}$. Deux diagrammes $d=\left(f_{1}, f_{2}\right)$, $d^{\prime}=\left(f_{1}^{\prime}, f_{2}^{\prime}\right)$ sont holomorphiquement (resp. formellement) conjugués s'il existe des éléments $h, \ell_{1}, \ell_{2}$ de $\mathbb{D}$ (resp. $\left.\widehat{\mathbb{D}}\right)$ tels que :

$$
f_{j} \circ h=\ell_{j} \circ f_{j}^{\prime} \quad \text { pour } i=1,2 .
$$

Il est clair que les $\ell_{i}$ appartiennent à $\mathbb{D}$ dès que $h$ appartient à $\mathbb{D}$.

Le groupe d'invariance $G(d)$ de $d=\left(f_{1}, f_{2}\right)$ est le sous-groupe de $\mathbb{D}$ engendré par les $g$ tels que $f_{j} \circ g=f_{j}$ pour $j=1$ ou $j=2$. Ce groupe est engendré par deux éléments périodiques $g_{1}, g_{2}$ de période $\nu\left(f_{1}\right), \nu\left(f_{2}\right)$ où $\nu\left(f_{j}\right)$ désigne l'ordre de $f_{j}$. Nous dirons que $d$ est abélien si $G(d)$ est un groupe abélien.

Assertion 1. - Un diagramme abélien $d$ est holomorphiquement conjugué à un diagramme monomial $\left(x^{\nu_{1}}, x^{\nu_{2}}\right)$.

Preuve. - Le groupe $G(d)$ étant abélien, engendré par deux éléments périodiques $g_{1}, g_{2}$ de périodes $\nu_{1}, \nu_{2}$, est un groupe fini. Il est conjugué à un groupe de rotations périodiques. En particulier, il existe $h \in \mathbb{D}$ tel que :

$$
g_{j} \circ h(x)=h\left(e^{2 i \pi / \nu_{j}} \cdot x\right) .
$$

Ainsi, $f_{j} \circ h$ est invariant par la rotation d'angle $e^{2 i \pi / \nu_{j}}$ et est d'ordre $\nu_{j}$; il s'écrit :

$$
f_{j} \circ h(x)=\ell_{j}\left(x^{\nu_{j}}\right) \quad \text { avec } \quad \ell_{j} \in \mathbb{D} .
$$

Assertion $2[\mathrm{D}]$. - $\mathrm{Si} d, d^{\prime}$ sont holomorphiquement (resp. formellement) conjugués, alors $G(d), G\left(d^{\prime}\right)$ le sont aussi.

TOME $116-1988-\mathrm{N}^{\circ} 4$ 
Soient $p, n_{1}, n_{2}$ des entiers tels que les $n_{i}$ soient impairs et $0<n_{i}<2 p$. Notons $q_{i}$ le ppcm de $p$ et $n_{i}, s_{i}=q_{i / n_{i}}$ et

$$
d\left(n_{1}, n_{2}, p\right)=\left(x^{2 s_{1}}, \frac{x^{2 s_{2}}}{\left(1-x^{p}\right)^{2 s_{2 / p}}}\right)
$$

On remarquera que $G\left(d\left(n_{1}, n_{2}, p\right)\right)$ est holomorphiquement conjugué au groupe $G_{n_{1}, n_{2}, p}$ de la Remarque 1.1. Le théorème suivant généralise un résultat de VORONIN [V] :

THÉORÈme 2. - Deux diagrammes $d, d^{\prime}$ qui sont formellement conjugués le sont holomorphiquement lorsque $d, d^{\prime}$ ne sont pas formellement conjugués à l'un des $d\left(n_{1}, n_{2}, p\right)$ avec $n_{1}, n_{2}$ impairs.

Preuve. - L'Assertion 1 prouve le théorème lorsque $d$ est abélien. Si $d$ n'est pas abélien et n'est pas conjugué formellement à l'un des $d\left(n_{1}, n_{2}, p\right)$ on note que $G(d)$ n'est pas formellement conjugué à l'un des $G_{\omega, p}$. Le théorème est alors une conséquence de l'Assertion 2 et de la Remarque 1.1.

On déduit du théorème que $d=\left(f_{1}, f_{2}\right)$ n'est pas formellement conjugué à un $d\left(n_{1}, n_{2}, p\right)$ si l'un des $\nu\left(f_{i}\right)$ est impair (Corollaire 1.1). Le résultat de Voronin concerne les diagrammes génériques du type $\left(x^{2}, x^{2}+\cdots\right)$. Ils sont formellement conjugués à $d(1,1,1)$; leur espace de modules formels s'identifie à $G_{-1,1}^{\text {For }}$.

Remarque 1. - On aurait pu considérer des diagrammes à $n$ flèches $n>2$, génériques; $G(d)$ est alors engendré par plus de deux éléments. Par suite, "génériquement" $d$ et $d^{\prime}$ sont holomorphiquement conjugués si et seulement si ils le sont formellement.

\section{Conjugaisons holomorphes et formelles d'équations différentielles $y d y+\cdots=0$}

1. Préliminaires et résultats. - Soit $\Lambda$ l'ensemble des germes en $0 \in \mathbb{C}^{2}$ de formes de Pfaff $\Omega=a d x+b d y$ à singularité algèbriquement isolée. Deux telles formes $\Omega, \Omega^{\prime}$ sont holomorphiquement (resp. formellement) conjuguées s'il existe un élément $\Phi$ de $\mathbb{C}\{x, y\}^{2}$ (resp. $\mathbb{C}[[x, y]]^{2}$ ) tel que

$$
\Phi^{*} \Omega \wedge \Omega^{\prime}=0 \text { et } D \emptyset(0) \in \mathrm{GL}(2, \mathbb{C}) .
$$

$L$ 'espace des modules formels de $\Omega$ est le quotient $\Omega^{\text {For }}$ de l'ensemble des $\Omega^{\prime}$ formellement conjugués à $\Omega$ par la relation "conjugaison holomorphe".

Une forme $\Omega$ est dégénérée si le jet d'ordre $1, D X(0)$, du champ dual $X_{\Omega}=b(\partial / \partial x)-a(\partial / \partial y)$ est nilpotent. Soit $\Sigma_{1}$ l'ensemble des $\Omega \in \Lambda$ qui 
sont dégénérées et dont le 1-jet n'est pas nul; modulo un changement de coordonnées linéaires il s'écrit $J^{1} \Omega=y d y$.

Assertion [T]. - Une forme $\Omega$ de $\Sigma_{1}$ est formellement conjuguée à une forme du type :

$$
\Omega_{n, p}=d\left(y^{2}+x^{n}\right)+x^{p} U(x) d y
$$

où $n \geq 3, p \geq 2$ sont des entiers et $U \in \mathbb{C}[[x]], U(0) \neq 0$.

Nous dirons, abusivement (voir paragraphe 2) que $\Omega_{n, p}$ est une forme normale de $\Omega$.

THÉORÈme 3. - Soit $\Omega$ un élément de $\Sigma_{1}$ ayant une forme normale de type $\Omega_{n, p}$ avec $2 p>n$. Son espace de module $\Omega^{\mathrm{For}}$ est en général trivial; s'il ne l'est pas $n$ est pair et il existe $\lambda \in \mathbb{C}, q \in N^{*}$ uniques tels que $\Omega^{\text {For }}$ s'identifie à l'espace des modules formels $A_{n, q, \lambda}^{\text {For }} d u$ sous-groupe (abélien) $A_{n, q, \lambda}$ de $\mathbb{D}$ engendré par:

$$
f(x)=e^{4 i(\pi / n)} x, \quad g(x)=e^{2 i(\pi / n)} \exp X_{n q, \lambda}(x) .
$$

De la même façon que pour les groupes $G_{\omega, p}$, l'espace $A_{n, q, \lambda}^{\text {For }}$ s'identifie aux cocycles d'ECALle des $\check{g} \in \mathbb{D}$ qui sont formellement conjugués à $g$ et qui sont équivariants par $f$. Ce théorème est à rapprocher des résultats classiques sur $\Omega^{\text {For }}$ pour une forme $\Omega$ non dégénérée. Dans ce cas, le type de $\Omega^{\text {For }}$ dépend essentiellement de l'exposant caractéristique de $\Omega$, $\mu(\Omega)=-\lambda^{\prime} / \lambda^{\prime \prime}$ où $\lambda^{\prime}, \lambda^{\prime \prime} \neq 0$ sont les valeurs propres du champ $X_{\Omega}$ :

i) $\Omega^{\text {For }}$ est trivial si $\mu(\Omega) \in \mathbb{C}-\mathbb{R}^{-}$(Poincare-Dulac, $[\mathrm{P}],[\mathrm{Du}]$ ).

ii) $\Omega^{\text {For }}$ est trivial si $\mu(\Omega) \in \mathbb{R}^{+}-\mathbb{Q}$ vérifie une condition diophantienne (SIEGEL $[\mathrm{S}]$ ).

iii) $\Omega^{\text {For }}$ s'identifie à l'espace (à un sous-espace si $\mu(\Omega)=0$ ) des modules d'un germe $h \in \mathbb{D}$ si $\mu(\Omega)=p / q, p, q \in \mathbb{N}$ avec $h^{\prime}(0)=e^{2 i \pi(p / q)}$. (J. Ecalle $\left[\mathrm{E}_{1}\right],\left[\mathrm{E}_{2}\right]$, J. Martinet et J.-P. Ramis $\left.\left[\mathrm{MR}_{i}\right]\right)$.

Cette identification est explicitée par l'assertion suivante, fondamentale dans la preuve du théorème.

Assertion $2\left[\mathrm{M}, \mathrm{R}_{1}\right]$, [Mo]. - Soit $\Omega$ une forme non dégénérée d'exposant caractéristique $\mu \in \mathbb{R}^{+}$. L'équation différentielle $\Omega=0$ possède deux courbes intégrales $L^{\prime}, L^{\prime \prime}$ lisses (ses séparatrices) tangentes aux directions propres correspondant à $\lambda^{\prime}, \lambda^{\prime \prime}$. La monodromie $h \in \mathbb{D}$ de $L^{\prime}$ caractérise $\Omega$ : deux formes $\Omega_{1}, \Omega_{2}$ d'exposants caractéristiques $\mu_{1}=$ $\mu_{2}=\mu$ sont holomorphiquement (resp. formellement) conjuguées si et seulement si les monodromies $h_{1}, h_{2}$ de $L_{1}^{\prime}=L_{2}^{\prime}$ sont holomorphiquement (resp. formellement) conjuguées. En particulier, si $\mu=p / q \in \mathbb{Q}^{+}, \Omega^{\text {For }}$

$$
\text { TOME } 116-1988-\mathrm{N}^{\circ} 4
$$


est trivial si et seulement si $h$ est périodique. Dans ce cas $\Omega$ possède une intégrale première holomorphe du type $x^{p} y^{q}$ dans un bon système de coordonnées.

2. Définition de $\Sigma_{1}^{n}$. - Soit $\Omega_{n, p}$ une forme normale d'un $\Omega$ appartenant à $\Sigma_{1}$. L'entier $n$ est un invariant formel de $\Omega$; en effet, la dimension de l'espace vectoriel $\mathbb{C}[[x, y]] / I(\Omega)$, où $I(\Omega)$ est l'idéal engendré par $a, b(\Omega=a d x+b d y)$, est un invariant formel et on a :

$$
\operatorname{dim} \mathbb{C}[[x, y]] / I(\Omega)=\operatorname{dim} \mathbb{C}[[x, y]] / I\left(\Omega_{n, p}\right)=n+1
$$

Par contre $p$ n'est pas un invariant formel. Cependant, la condition $2 p>n$ dans l'énoncé du théorème est invariante par conjugaison formelle. Nous noterons $\Sigma_{1}^{n}$ l'ensemble des $\Omega$ de $\Sigma_{1}$ qui possèdent une forme normale de type $\Omega_{n, p}$ avec $2 p>n$.

Dans la suite, nous choisissons des coordonnées $(x, y)$ de $\left(\mathbb{C}^{2}, 0\right)$ de telle façon que le jet d'ordre $2 n$ d'un $\Omega \in \Sigma_{1}^{n}$ soit précisément une forme de type $\Omega_{n, p}$. L'équation différentielle $\Omega=0$ apparait alors comme une perturbation d'ordre $p>n / 2$ de l'équation hamiltonienne $d\left(y^{2}+x^{n}\right)=$ 0 . Ceci est précisé par la Proposition 1 ci-dessous et le résultat du THÉORÈmE 4 peut s'énoncer :

Des perturbations d'ordre $p>n / 2$ de l'équation $d\left(y^{2}+x^{n}\right)=0$ qui sont formellement conjuguées sont en général holomorphiquement conjuguées.

Par contre, la classification des $\Omega \in \Sigma_{1}$ qui ont une forme normale $\Omega_{n, p}$ avec $p \leq n / 2$ est "zoologique". Elle dépend d'un certain nombre de paramètres, les coefficients des $x^{i}$ dans $x^{p} U(x)$ pour $i \leq n / 2$. La preuve $\mathrm{du}$ théorème repose sur le concept d'holonomie projective $[\mathrm{M}, \mathrm{M}]$ que nous définissons dans le paragraphe ci-dessous.

3. Résolution minimale des éléments de $\Sigma_{1}^{n}$. - Une résolution d'une forme $\Omega \in \Lambda$ est un morphisme algébrique

$$
\pi:(M, D) \longrightarrow\left(\mathbb{C}^{2}, 0\right), \quad \pi^{-1}(0)=D
$$

obtenu par composition de $n_{\pi}$ éclatements ponctuels qui vérifie la propriété suivante : en chaque point $m$ de $D$ il existe une équation locale $f_{m}=0$ de $D$ telle que le germe en $m, \widetilde{\Omega}_{m}$ de l'éclaté-divisé $\pi^{*}(\Omega) / f_{m}$ soit non dégénéré. $\pi$ est dite minimale si pour toute autre résolution $\pi^{\prime}$ de $\Omega, n_{\pi^{\prime}}>n_{\pi}$; nous la notons $\pi_{\Omega}$. Enfin nous dirons que $\Omega, \Omega^{\prime}$ ont la même résolution minimale à l'ordre $\nu$ si $\pi_{\Omega}=\pi_{\Omega^{\prime}}$ et si les éclatés divisés de $\Omega, \Omega^{\prime}$ ont les mêmes points singuliers $m_{1}, m_{2}, \ldots, m_{r}$ et les jets d'ordre $\nu \operatorname{des} \widetilde{\Omega}_{m_{k}}$ et $\widetilde{\Omega}_{m_{k}}^{\prime}$ sont colinéaires. 
Un élément de $\Sigma_{1}^{n}$ étant formellement conjugué à une forme de type $\Omega_{n, p}$, nous choisissons les coordonnées $(x, y)$ de $\left(\mathbb{C}^{2}, 0\right)$ de telle façon que son jet d'ordre $2 n$ soit une forme de type $\Omega_{n, p}$. Cette condition est supposée satisfaite dans toute la suite.

Proposition 1. - Un élément $\Omega$ de $\Sigma_{1}^{n}$ a la même résolution $\grave{a}$ l'ordre 1 que $\Omega_{n}=d\left(y^{2}+x^{n}\right)$. Plus précisément si :

i) $n=2 n^{\prime}$, $D$ est une chaine de $n^{\prime}$ droites projectives $D_{k}$, avec $k=1,2, \ldots, n^{\prime}$ qui se coupent aux points $m_{k}=D_{k} \cap D_{k+1}$ pour $k=1,2, \ldots, n^{\prime}-1$. Les éclatés divisés de $\Omega$ ont pour singularités ces $\left(n^{\prime}-1\right)$ points de croisement et deux points $m^{\prime}, m^{\prime \prime}$ de $D_{n^{\prime}}$ (voir figure 3.1.a).

ii) $n=2 n^{\prime}+1, D$ est une chaine de $\left(n^{\prime}+2\right)$-droites projectives $D_{k}$, $k=1,2, \ldots, n^{\prime}+2$ qui se coupent aux points $m_{k}=D_{k} \cap D_{k+1}$ pour $k=1,2, \ldots, n^{\prime}+1$. Les éclatés divisés de $\Omega$ ont pour singularités ces $\left(n^{\prime}+1\right)$ points et un point $m$ de $D_{n^{\prime}+1}$. (Figure 3.1.b).

Preuve pour $n=2 n^{\prime}$. - La résolution minimale de $\Omega_{n}$ est le composé $\pi$ de $n^{\prime}$-éclatements qui s'écrivent :

$$
y=x_{1} x ; \quad x_{1}=x_{2} x ; \ldots ; \quad x_{n^{\prime}-2}=x_{n^{\prime}-1} x ; \quad x_{n^{\prime}-1}=t \cdot x .
$$

Les $D_{k}$ ont pour équation $x=0$ dans les cartes $\left(x, x_{k}\right)$ pour $k<n$. Dans la carte $(t, x)$ on a :

$$
\begin{aligned}
\pi(t, x) & =\left(x, t x^{n^{\prime}}\right), D_{n^{\prime}}=\{x=0\} \\
\pi^{*} \Omega_{n} & =d\left(x^{n}\left(1+t^{2}\right)\right)
\end{aligned}
$$

compte-tenu de l'inégalité $2 p>n$ on vérifie aisément que $\pi$ est une résolution de $\Omega$ et que les éclatés divisés de $\Omega$ et $\Omega_{n}$ ont les mêmes jets d'ordre 1 aux points de croisement ainsi qu'aux points singuliers $m^{\prime}, m^{\prime \prime} \in D_{n^{\prime}}$ de coordonnées respectives $(i, 0),(-i, 0)$ dans la carte $(t, x)$.

Preuve pour $n=2 n^{\prime}+1$. - La résolution minimale $\pi$ de $\Omega_{n}$ est le composé de $\left(n^{\prime}+2\right)$-éclatements qui s'écrivent

$$
y=x_{1} x ; \ldots ; \quad x_{n^{\prime}-1}=x_{n^{\prime}} x ; \quad x=u x_{n^{\prime}} ; \quad x_{n^{\prime}}=u t .
$$

Les $D_{k}$ ont pour équation $x=0$ dans les cartes $\left(x, x_{k}\right)$ pour $k<n^{\prime}$; dans la carte $(t, u)$ les projectifs $D_{n^{\prime}}, D_{n^{\prime}+1}$ ont respectivement pour équation $t=0, u=0$ et on a

$$
\begin{aligned}
\pi(t, u) & =\left(u^{2} t, u^{n} t^{n+1}\right) \\
\pi^{*}\left(\Omega_{n}\right) & =d\left(u^{2 n} t^{n}(1+t)\right) .
\end{aligned}
$$

TOME $116-1988-\mathrm{N}^{\circ} 4$ 
Comme dans le cas précédent, on vérifie que $\pi$ est une résolution minimale de $\Omega$ et que les éclatés divisés de $\Omega$ et $\Omega_{n}$ ont les mêmes jets d'ordre 1 aux points de croisements des $D_{k}$ ainsi qu'au point singulier $m \in \mathbb{D}_{n^{\prime}+1}$, de coordonnées $(-1,0)$ dans la carte $(t, u)$.

4. Holonomie projective des éléments de $\Sigma_{1}^{n}$ - - Soit, comme dans le paragraphe précédent, $\Omega$ un élément de $\Sigma_{1}^{n}$ dont le $2 n$-jet est du type $\Omega_{n, p}$ avec $2 p>n$ et soit $\pi:(M, D) \rightarrow\left(\mathbb{C}^{2}, 0\right)$ sa résolution minimale.

L'équation $\Omega=0$ définit un feuilletage singulier holomorphe $F_{\Omega}$ au voisinage de 0 . Son image réciproque par $\pi, \pi^{*} F_{\Omega}$ est un feuilletage singulier défini sur un voisinage de $D$ par les éclatés divisés de $\Omega$. Ainsi, il a pour points singuliers :

les points de croisements $m_{k}$ des $D_{k}$;

les points $m^{\prime}, m^{\prime \prime}$ si $n$ est pair, le point $m$ si $n$ est impair.

La restriction de $\pi^{*} F_{\Omega}$ au complémentaire de ces points est un feuilletage (régulier) encore noté $\pi^{*} F_{\Omega}$; le complémentaire dans chaque $D_{k}$ des points singuliers est une feuille $L_{k}$ de $\pi^{*} F_{\Omega}$. On note $H_{k}$ la représentation d'holonomie de $L_{k}$ :

$$
H_{k}: \pi_{1}\left(L_{k}\right) \longrightarrow \mathbb{D} \text {. }
$$

Puisque $L_{1}=D_{1}-\left\{m_{1}\right\}$ (resp. $L_{n^{\prime}+1}=D_{n^{\prime}+1}-m_{n}$ si $n$ est impair), $H_{1}$ (resp. $H_{m_{n^{\prime}+1}}$ ) a pour image $1_{\mathbb{C}}$ et d'après l'Assertion $2, \Omega_{\tilde{m}_{1}}$ (resp. $\widetilde{\Omega}_{m_{n^{\prime}+1}}$ ) possède une intégrale première. La deuxième séparatrice $L_{2}$ en $m_{1}$ a ainsi une holonomie périodique. Puisque $L_{2}=D_{2}-\left\{m_{1}, m_{2}\right\}$ cette holonomie est aussi celle de $L_{2}$ en tant que séparatrice de $\widetilde{\Omega}_{m_{2}}$. D'après l'Assertion 2, $\widetilde{\Omega}_{m_{2}}$ a une intégrale première. Par une induction évidente, on en déduit, compte-tenu de la proposition précédente que si :

i) $n$ est pair. Les $\widetilde{\Omega}_{m_{k}}$ possèdent des intégrales premières pour $k=$ $1,2, \ldots, n^{\prime}-1$. Nous appelons holonomie projective de $\Omega$ la représentation d'holonomie

$$
H_{n^{\prime}}=H_{\Omega}: \pi_{1}\left(L_{n^{\prime}}, 0\right) \longrightarrow \mathbb{D}
$$

où 0 est le point de coordonnées $(0,0)$ dans la carte $(t, x)$. Soient $\gamma, \gamma_{1}, \gamma_{2}$ des éléments de $\pi_{1}\left(L_{n^{\prime}}, 0\right)$ tels que $m_{n^{\prime}-1}, m^{\prime}, m^{\prime \prime}$ soient respectivement des points d'indice 1 pour ces lacets (Figure 3.2.a) et soient

$$
f=H_{\Omega}\left(\gamma^{-1}\right), \quad f_{j}=H_{\Omega}\left(\gamma_{j}\right) \quad \text { pour } \quad j=1,2 .
$$

Alors, on a :

$$
\begin{aligned}
f^{\prime}(0) & =e^{4 i \pi / n}, \quad f_{j}^{\prime}(0)=e^{2 i \pi / n} \quad \text { pour } \quad j=1,2 \\
f & =f_{2} \circ f_{1}, \quad f^{n}=1_{\mathbb{C}} .
\end{aligned}
$$


ii) $n$ est impair. Les $\widetilde{\Omega}_{m_{k}}$ possèdent des intégrales premières pour $k=$ $1,2, \ldots, n^{\prime}+1$. Nous appelons holonomie projective de $\Omega$ la représentation d'holonomie

$$
H_{n^{\prime}+1}=H_{\Omega}: \pi_{1}\left(L_{n^{\prime}+1}, 0\right) \longrightarrow \mathrm{D}
$$

où 0 est le point $(0,0)$ dans la carte $(t, u)$. Soient $\gamma, \gamma_{1}, \gamma_{2}$ des éléments de $\pi_{1}\left(L_{n^{\prime}+1}, 0\right)$ tels que $m, m_{n^{\prime}}, m_{n^{\prime}+1}$ soient respectivement des points d'indice 1 pour ces lacets (Figure 3.2.b) et soient

$$
f=H_{\Omega}(\gamma), \quad f_{i}=H_{\Omega}\left(\gamma_{i}\right) \text { pour } \quad i=1,2 .
$$

Alors on a :

$$
\begin{aligned}
f^{\prime}(0) & =e^{-i \pi / n}, \quad f_{1}^{\prime}(0)=e^{i \pi}, \quad f_{2}^{\prime}(0)=e^{i \pi(n-1 / n)} \\
f & =f_{2} \circ f_{1}, \quad f_{1}^{2}=f_{2}^{n}=1_{\mathbf{C}} .
\end{aligned}
$$

Dans les deux cas, l'image de $H_{\Omega}$ est notée $G_{\Omega} ; G_{\Omega}$ est le groupe d'holonomie de $\Omega$.
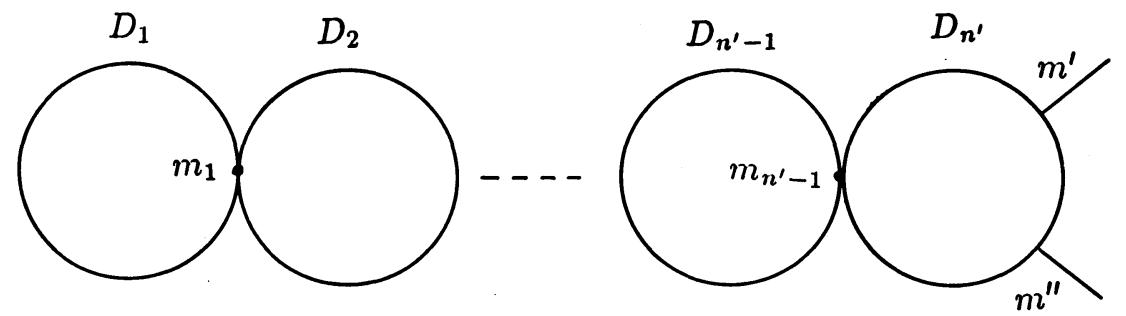

Fig. III 1 a

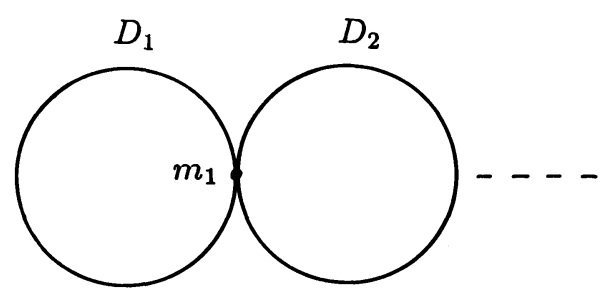

Fig. III $1 \mathrm{~b}$

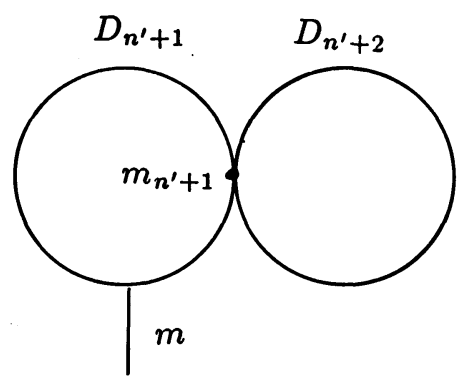

TOME $116-1988-\mathrm{N}^{\circ} 4$ 


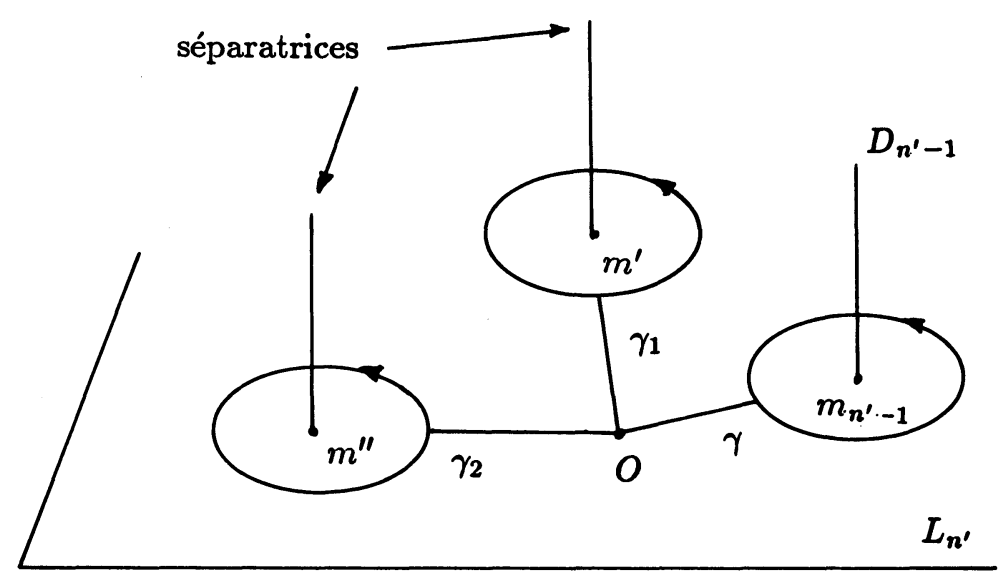

Fig. III 2 a

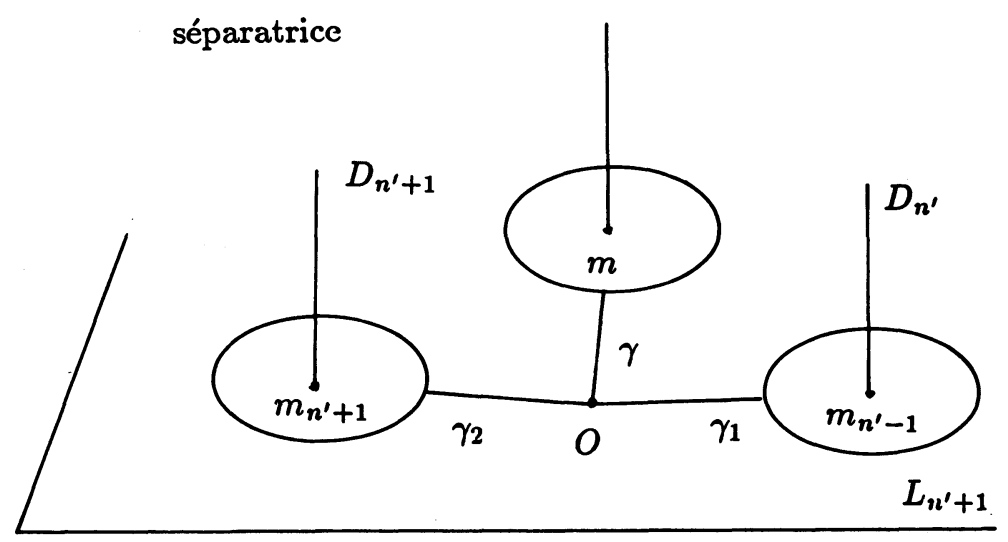

Fig. III 2 b

BULLETIN DE LA SOCIÉTÉ MATHÉMATIQUE DE FRANCE 
5. Démonstration du théorème. - Nous allons tout d'abord montrer que c'est une conséquence de la proposition suivante.

Proposition 4 (voir $\left[\mathrm{M}_{0}\right]$ pour $n=3$ ). - Deux formes $\Omega, \Omega^{\prime}$ de $\Sigma_{1}^{n}$ sont holomorphiquement (resp. formellement) conjuguées si et seulement si leurs groupes d'holonomie $G_{\Omega}, G_{\Omega^{\prime}}$ sont holomorphiquement (resp. formellement conjugués).

Soient $\Omega, \Omega^{\prime}$ deux éléments de $\Sigma_{1}^{n}$ formellement conjugués. Leurs groupes d'holonomie sont formellement conjugués. Nous allons montrer tout d'abord qu'ils le sont holomorphiquement dans les 3 cas suivants :

i) $n$ est impair et $G_{\Omega}$ n'est pas abélien. - $G_{\Omega}$ est engendré par $f_{1}, f_{2}$ qui sont périodiques et $f_{2}$ est de période impaire. D'après le Corollaire $1.1, G_{\Omega}$ et $G_{\Omega^{\prime}}$ sont holomorphiquement conjugués.

ii) $n$ est impair et $G_{\Omega}$ est abélien. - $G_{\Omega}$ et $G_{\Omega^{\prime}}$ sont holomorphiquement conjugués au groupe engendré par la rotation $x \rightarrow e^{i \pi / n} x$.

iii) $n$ est pair et $G_{\Omega}$ n'est pas abélien. - D'après le THÉORÈmE 1 il suffit de montrer que $G_{\Omega}$ n'est pas formellement conjugué à un groupe $G_{\omega, p}$. Si tel était le cas, modulo une conjugaison formelle $G_{\Omega}$ serait engendré par :

$$
f_{j}=e^{2 i \pi / n} \cdot \frac{x}{\sqrt[p]{1-p k_{j} x^{p}}} \text { avec } k_{j} \in \mathbb{Z}, j=1,2 .
$$

On vérifie que nécessairement $e^{2 i \pi p / n}=-1\left(\operatorname{car} \omega^{p}=-1\right)$ ainsi que :

$$
f=f_{2} \circ f_{1}=e^{4 i \pi / n} \cdot \frac{x}{\sqrt[p]{1-p\left(k_{1}+k_{2} e^{(2 i \pi / n)^{p}}\right) x^{p}}} \cdot
$$

Puisque $f$ est périodique de période paire, d'après la Remarque 1.1,

$$
k_{1}+k_{2} e^{(2 i \pi / n)^{p}}=0 .
$$

La condition $e^{2 i \pi p / n}=-1$, implique $k_{1}=k_{2}$ et ainsi on aurait $f_{1}=f_{2}$, et $G_{\Omega}$ serait abélien.

Ainsi, il peut apparaitre un espace de module non trivial seulement si :

iv) $n$ est pair et $G_{\Omega}$ est abélien. - Si $f_{1}$ et $f_{2}$ sont périodiques alors $G_{\Omega}$ est holomorphiquement conjugué au groupe engendré par la rotation $x \rightarrow e^{2 i \pi / n} \cdot x$; ici encore il n'y a pas de modules. $G_{\Omega}$ étant abélien et $f_{1} \circ f_{2}$ périodique, il nous reste à étudier le cas où $f_{1}$ et $f_{2}$ ne sont pas périodiques. Montrons qu'alors $G_{\Omega}$ est formellement conjugué à un groupe $A_{n, q, \lambda}$. Puisque $f_{1}^{\prime}(0)=f_{2}^{\prime}(0)=e^{2 i \pi / n}, f_{2} \circ f_{1}^{-1}$ est tangent à $1_{\mathbb{C}}$ et $G_{\Omega} \cap \mathbb{D}_{1}$ est monogène. D'après la Proposition 1.2, à une conjugaison formelle près $G_{\Omega}$ est engendré par les

$$
f_{j}=\omega \exp t_{j} X_{p, \lambda} \quad j=1,2
$$

TOME $116-1988-\mathrm{N}^{\circ} 4$ 
avec $\omega=e^{2 i \pi / n}, \omega^{p}=1, p=q . n, q \in \mathbb{N}^{*}$. En écrivant que $f=$ $f_{1} \circ f_{2}=\omega^{2} \exp \left(t_{1}+t_{2}\right) X_{p, \lambda}$ est périodique, on obtient $t_{1}+t_{2}=0$ et $f(x)=\omega^{2} x . G_{\Omega}$ est à conjugaison formelle près engendré par $x \mapsto e^{4 i \pi / n}$ et $x \mapsto e^{2 i \pi / n} \exp X_{n q, \lambda}$.

Remarque 1. - Soit $\Omega$ un élément de $\Sigma_{1}^{n}$. Un argument de [M,M] montre que $G_{\Omega}$ est abélien et est engendré par des éléments périodiques si et seulement si $\Omega$ possède une intégrale première holomorphe. Le THÉORÈmE 3 peut encore s'énoncer : deux éléments $\Omega, \Omega^{\prime}$ de $\Sigma_{1}^{n}$ qui sont formellement conjugués le sont holomorphiquement si et seulement si une des conditions suivantes est vérifiée :

$G_{\Omega}$ n'est pas abélien,

$G_{\Omega}$ est abélien et $\Omega$ possède une intégrale première holomorphe ou même formelle.

Remarque 2. - Soit $G$ un sous-groupe de $\mathbb{D}$ qui est engendré par deux éléments $f_{1}, f_{2}$ tels que :

$$
f_{1}^{\prime}(0)=f_{2}^{\prime}(0)=e^{i \pi / n^{\prime}}\left(f_{2} \circ f_{1}\right)^{n^{\prime}}=1_{\mathbb{C}} .
$$

Il existe un élément $\Omega \in \Sigma_{1}^{2 n^{\prime}}$ tel que $G_{\Omega}$ soit le sous-groupe de $\mathbb{D}$ engendré $\operatorname{par}\left(f_{1}, f_{2}\right)$. En effet, les éléments $f_{j} \in \mathbb{D}$ (à partie linéaire périodique) sont réalisables comme difféomorphismes de monodromie d'équations différentielles $\Omega_{i}$ non dégénérées de 1 -jet $n^{\prime} y d x+x d y\left(\left[\mathrm{M}, \mathrm{R}_{2}\right]\right)$.

En utilisant le théorème de Grauert classifiant les voisinages tubulaires de courbes lisses à classe de CHERN négative, on construit à partir des $\Omega_{i}$ un feuilletage $F$ d'une variété $(M, D)$ qui correspond à la résolution minimale d'une forme $\Omega \in \Sigma_{1}^{n}$ avec $G_{\Omega}=\left(f_{1}, f_{2}\right)$ (cf. [L] pour un problème analogue). En particulier, il est possible de réaliser comme $G_{\Omega}$, $\Omega \in \Sigma_{1}^{n}$, tous les groupes formellement conjugués à un groupe $A_{n, q, \lambda}$ et on a évidemment

$$
\Omega^{\text {For }} \equiv F_{\Omega}^{\text {For }} \equiv A_{n, q, \lambda}^{\text {For }} .
$$

Lorsque $\lambda=0$, de tels $\Omega$ ont une équation algèbrique. Par exemple :

$$
\Omega=d\left(y^{2}+x^{4}\right)+4 i \pi x\left(y^{2}+x^{4}\right)(2 y d x-x d y)
$$

réalise le groupe $A_{4,1,0}$. Ceci se montre en remarquant que si $G_{\Omega}=A_{n, q, 0}$, $\pi^{*} \Omega=0$ est, au voisinage de $D_{n^{\prime}}$, conjuguée à un "revêtement" d'une équation de Ricatti.

Preuve de la Proposition 4. - Elle se scinde en deux parties:

1) La conjugaison des formes implique la conjugaison des holonomies. Soient $\Omega, \Omega^{\prime}$ deux éléments de $\Sigma_{1}^{n}$ formellement conjugués par $\Phi \in$ 
$\mathbb{C}[[x, y]]^{2}$. On peut toujours supposer que $\Phi_{N}=J^{N} \Phi$ est $1_{\mathbb{C}}$ pour $N$ grand et ainsi $\Omega$ et $\Omega^{\prime}$ ont la même résolution minimale, $\pi:(M, D) \rightarrow\left(\mathbb{C}^{2}, 0\right)$. Nous notons $H_{\Omega}, H_{\Omega^{\prime}}$ leur représentation d'holonomie évaluée sur la transversale $\{t=0\}$.

Lorsque $\Phi$ est holomorphe, tangent à $1_{C}$ à un ordre grand, il se relève en un difféomorphisme $\tilde{\Phi}:(M, D) \circlearrowleft$ qui applique $\pi^{*} F_{\Omega}$ sur $\pi^{*} F_{\Omega^{\prime}}$ et tel que $\tilde{\Phi} / D=1_{D}$. Sa restriction à $\{t=0\}$ conjugue $H_{\Omega}$ et la représentation d'holonomie de $\Omega^{\prime}$ évaluée sur $\tilde{\Phi}(\{t=0\})$. Ainsi cette dernière est holomorphiquement conjuguée à $H_{\Omega^{\prime}}$.

Lorsque $\Phi$ est formel, son jet d'ordre $k, \Phi_{k}$ se relève en $\tilde{\Phi}_{k}:(M, D) \leftrightharpoons$ qui applique $\pi^{*} F_{\Omega}$ sur $\pi^{*} F_{\Omega_{k}^{\prime}}$ avec $\Omega_{k}^{\prime}=\Phi_{k}^{*} \Omega$; les représentations $H_{\Omega}$ et $H_{\Omega^{\prime}}$ sont conjuguées. D'autre part, il existe une suite $\ell(k)$ tendant vers l'infini telle que :

$$
\pi^{*}\left(\Omega_{k}^{\prime}\right) \wedge \pi^{*}\left(\Omega^{\prime}\right)=0 \quad\left(\bmod x^{\ell(k)}\right)
$$

où $x=0$ est l'équation de $D_{n^{\prime}}$ si $n$ est pair, de $D_{n^{\prime}+1}$ si $n$ est impair. Ainsi $H_{\Omega_{k}^{\prime}}$ et $H_{\Omega^{\prime}}$ sont conjugués modulo $x^{\ell(k)}$ par un difféomorphisme polynomial $\varphi_{k}$. La famille $\varphi_{k}$ est filtrée; sa limite $\varphi$ conjugue formellement $H_{\Omega^{\prime}}$ et $\lim _{k \rightarrow \infty} H_{\Omega_{k}^{\prime}}$.

2) La conjugaison des holonomies implique la conjugaison des formes. Ce point est démontré pour $n=3$ dans $\left[\mathrm{M}_{0}\right]$; la démonstration lorsque $n$ est impair est essentiellement la même. Dans tous les cas elle repose sur l'assertion suivante :

Assertion 1. - Soit $\Omega$ un élément de $\Sigma_{1}^{n}$; il existe des coordonnées $(x, y)$ de $\mathbb{C}^{2}, 0$ dans lesquelles $\Omega$ s'écrit à unité multiplicative près :

$$
\Omega=d\left(y^{2}+x^{n}\right)+f(x, y)(n y d x-2 x d y), \quad \text { où } f \in \mathbb{C}\{x, y\} .
$$

Preuve. - Elle résulte du fait suivant : puisque $\Omega$ à la même résolution minimale que $d\left(y^{2}+x^{n}\right), \Omega$ possède une séparatrice $Z$ (ou deux lorsque $n$ est pair) tangente à l'ordre $n$ à $\left(y^{2}+x^{n}=0\right)$; dans un bon système de coordonnées $Z$ s'écrit $\left\{X^{2}+Y^{n}=0\right\}$. Par suite :

$$
\Omega \wedge d\left(X^{2}+Y^{n}\right)=\left(X^{2}+Y^{n}\right) H_{1} \cdot d X \wedge d Y, H_{1} \in \mathbb{C}\{X, Y\} .
$$

Comme $X^{2}+Y^{n}=0$ est une séparatrice de $2 Y d X-n X d Y$, on a :

$$
\Omega \wedge(n Y d X-2 X d Y)=\left(Y^{2}+X^{n}\right) H_{2} \cdot d X \wedge d Y, H_{2} \in \mathbb{C}\{X, Y\} .
$$

Puisque :

TOME $116-1988-\mathrm{N}^{\circ} 4$ 


$$
(n Y d X-2 X d Y) \wedge d\left(Y^{2}+X^{n}\right)=2 n\left(Y^{2}+X^{n}\right) d X \wedge d Y
$$

on obtient

$$
\Omega=-\frac{H_{2}}{2 n} d\left(Y^{2}+X^{n}\right)+\frac{H_{1}}{2 n}(n Y d X-2 X d Y) .
$$

On conclut en remarquant que $j^{1} \Omega=2 Y d Y$ implique $H_{2}(0) \neq 0$.

Il suffit de prouver que la conjugaison des holonomies implique la conjugaison des formes dans le cadre holomorphe. En effet, en raisonnant comme dans le paragraphe précédent sur les jets d'ordre $k$, le cas formel se déduit du cas holomorphe.

Soient $\Omega_{j}, j=1,2$ deux éléments de $\Sigma_{1}^{n}$ qui s'écrivent

$$
\Omega_{j}=d\left(y^{2}+x^{n}\right)+f_{j}(x, y) \cdot(2 x d y-n y d x) .
$$

Ces formes ayant la même résolution à l'ordre $1, \pi:(M, D) \rightarrow\left(\mathbb{C}^{2}, 0\right)$, nous supposons que leurs représentations d'holonomie $H_{\Omega_{j}}, j=1,2$ évaluées sur la même transversale $t=0$, sont liées par :

$$
H_{\Omega_{2}}=\varphi_{*} \circ H_{\Omega_{1}} \text { avec } \varphi \in \mathbb{D}_{1} .
$$

Nous allons montrer que $\varphi$ s'étend en un difféormorphisme $\tilde{\Phi}$ holomorphe de $(M, D)$ qui conjugue les $\pi^{*} F_{\Omega_{j}}$. Lorsque $n$ est impair les arguments de cette démonstration sont les mêmes que pour le cas $n=3$. Ce cas étant traité en détail dans $\left[\mathrm{M}_{0}\right]$, nous allons seulement prouver que pour $n$ pair $\varphi$ s'étend en $\tilde{\Phi}:(M, D) \zeta$. Soient $U_{j}, j=1,2$ des voisinages de 0 sur lesquels $\Omega_{j}$ possèdent un représentant holomorphe noté encore $\Omega_{j}$. Les feuilletages $\pi^{*} F_{\Omega_{j}}$ sont définis sur les $\pi^{-1}\left(U_{i}\right)$ par les $\pi^{*}\left(\Omega_{j}\right)$. D'après l'Assertion 1 , leurs restrictions à

$$
\pi^{-1}\left(U_{j}-\left\{x\left(y^{2}+x^{n}\right)=0\right\}\right)=V_{j}
$$

sont transverses à la fibration

$$
p: M-\{t=i, t=-i, \Delta\} \longrightarrow D_{n}^{\prime}-\left\{m^{\prime}, m^{\prime \prime}\right\}=L_{n^{\prime}}
$$

qui s'écrit $p(t, x)=x$ dans la carte $(t, x)$, où $\Delta$ est la droite $\pi^{-1}(\{x=0\})$.

L'argument de "relèvement des chemins" situés dans la feuille $L_{n^{\prime}}$ et d'origine le point $(0,0)$ permet d'étendre $\varphi$ en un difféomorphisme $\tilde{\Phi}$ holomorphe de $V_{1}$ dans $V_{2}$ qui conjugue les restrictions de $\pi^{*} F_{\Omega_{1}}$ et $\pi^{*} F_{\Omega_{2}}$ à ces ouverts (voir $[\mathrm{M}, \mathrm{M}]$ ). Les holonomies de $L_{n^{\prime}}$ (vu comme séparatrice de $\widetilde{\Omega}_{1, m^{\prime}}$ et $\left.\widetilde{\Omega}_{2, m^{\prime}}\right)$ étant holomorphiquement conjuguées l'argument de $\left[\mathrm{M}_{0}\right]$ ou $\left[\mathrm{M}, \mathrm{R}_{1}\right]$ prouve que $\varphi$ s'étend aussi de façon unique par relèvement des chemins sur un voisinage de $m^{\prime}$. 
Enfin, $\tilde{\Phi}$ se prolonge le long de $\Delta \cap V_{1}$ par le théorème d'Hartogs : en effet, les feuilletages $\pi^{*} F_{\Omega_{j}}$ sont transverses à $\Delta$ (comme les $\Omega_{j}$ le sont à $x=0$ ) et les applications composantes de $\Phi$ dans une carte qui contient $\Delta$ sont bornées par construction.

L'application $\tilde{\Phi}$ de $U_{1}-\{0\}$ dans $U_{2}-\{0\}$ définie par

$$
\Phi\left(\pi^{-1}(x, y)\right)=\tilde{\pi}(\Phi(x, y))
$$

est un difféomorphisme holomorphe qui se prolonge en un difféomorphisme holomorphe de $U_{1}$ sur $U_{2}$ appliquant $F_{\Omega_{1}}$ sur $F_{\Omega_{2}}$.

\section{Espaces des modules topologiques des sous-groupes de $\mathbb{H}$}

Rappelons que $\mathbb{H}$ est le sous-groupe de $\mathbb{D}$ constitué des germes en 0 d'homographies $h: x \rightarrow a x /(1+b x)$. La détermination de l'espace de modules topologiques d'un sous-groupe de $\mathbb{H}$ repose essentiellement sur le résultat "algèbrique" de la Proposition 1 ci-dessous et de son corollaire. C'est seulement dans le paragraphe 2 que nous aborderons les problèmes de conjugaison topologique.

\section{Sous-groupes de $\mathbb{D}$ isomorphes à un sous-groupe de $\mathbb{H}$.}

Proposition 1. - Soit $G$ un sous-groupe non abélien de $\mathbb{D}$ et soit $N$ la transformation normalisante d'un élément $h \neq 1_{\mathbb{C}}$ de $[G, G]$, $N_{*}(h)=\exp X_{p, \lambda}$. Si $G$ est isomorphe à un sous-groupe $H$ de $\mathbb{H}$ alors $\lambda=0$ et :

$$
N_{*}(G) \subset\left\{a \exp t X_{p, 0} ; a \in \mathbb{C}^{*}, t \in \mathbb{C}\right\} .
$$

De plus, si $H$ n'est pas homographiquement conjugué à $G_{-1,1}, N$ est convergente.

Corollaire 1. - Un sous-groupe non abélien $G$ de $\mathbb{D}$ topologiquement conjugué à un sous-groupe $H$ de $\mathbb{H}$ est holomorphiquement conjugué $\grave{a}$ un sous-groupe $H^{\prime}$ de $\mathbb{H}$ si $H$ n'est pas homographiquement conjugué à $G_{-1,1}$.

Ce corollaire est une conséquence immédiate de la proposition : l'homéomorphisme de conjugaison induit un isomorphisme de $G$ sur $H$. L'ordre de contact de $h$ avec $1_{\mathbb{C}}$ étant un invariant topologique, on a $p=1$; c'est-à-dire $N_{*}(G) \subset \mathbb{H}$.

On a une curieuse application de la proposition lorsque l'isomorphisme est induit par la projection canonique $j_{\mathrm{H}}$ de $\mathbb{D}$ sur $\mathbb{H}$ :

$$
j_{\mathrm{H}}(g)(x)=\frac{a x}{1+t x} \quad \text { si } g(x)=a x(1-t x+\cdots) .
$$

TOME $116-1988-\mathrm{N}^{\circ} 4$ 
CoRollaire 2. - Un sous-groupe non abélien $G$ de $\mathbb{D}$ est holomorphiquement conjugué à sa projection $j_{\mathbb{H}}(G)$ si la restriction de $j_{\mathbb{H}}$ à $G$ est injective et si $j_{\mathrm{H}}(G)$ n'est pas homographiquement conjugué à $G_{-1,1}$.

Démonstration de la Proposition 1. - Notons $H$ le groupe $j_{\mathrm{H}}(G)$, $N$ une transformation normalisante d'un élément non trivial $h$ de $[G, G]$, $N_{*}(h)=\exp \left(X_{p, \lambda}\right)$. La démonstration s'appuie sur la relation suivante :

$$
\left[g_{1 *}\left(h_{1}\right), h_{1}\right]=1_{\mathbb{C}}, \quad \text { si } \quad g_{1} \in H, h_{1} \in \mathbb{H} \cap D_{1} ;
$$

de plus $g_{1 *}\left(h_{1}\right)=h_{1}$ si et seulement si $g_{1} \in \mathbb{H} \cap D_{1}$. Il est clair que $h_{1}=j_{\mathrm{H}}(h)$ est un élément de $[H, H]$, donc de $H \cap D_{1}$. On en déduit que :

$$
\left[g_{*}(h), h\right]=1_{\mathbb{C}} \quad \text { si } \quad g \in G .
$$

Ainsi, $g_{*}(h)$ est un élément de $C_{1}(h)$. D'après l'assertion 0.1.6. :

$$
\left(N_{*} g\right)_{*} \exp X_{p, \lambda}=\exp t_{g} X_{p, \lambda} \quad \text { avec } \quad t_{g} \in \mathbb{C} .
$$

L'assertion 0.1.1. conduit alors à :

$$
\left(N_{*} g\right)_{*} X_{p, \lambda}=t_{g} X_{p, \lambda}
$$

Puisque $G$ n'est pas abélien il existe un élément $g \in G$ tel que $t_{g} \neq 1$. D'après l'assertion 0.1.4., $\lambda$ est nul et, pour tout élément $g \in G$, on a :

$$
N_{*}(g)=a_{g} \exp t_{g} X_{p, 0}, \quad a_{g}, t_{g} \in \mathbb{C} .
$$

Ceci prouve la Proposition 1 compte-tenu du Théorème 1.

2. Énoncé du théorème. - Deux sous-groupes $G, G^{\prime}$ de $\mathbb{D}$ sont topologiquement conjugués, $G \stackrel{\text { Top }}{\sim} G^{\prime}$, s'il existe un germe d'homéomorphisme $\Phi$ de $(\mathbb{C}, 0)$ qui préserve l'orientation tel que $\Phi_{*} G=G^{\prime}$. L'espace des modules topologiques de $G$ est l'ensemble

$$
G^{\mathrm{Top}}=\left\{\left[G^{\prime}\right]_{\mathbb{D}} ; G^{\mathrm{Top}} G^{\prime}, G^{\prime} \subset \mathbb{D}\right\}
$$

Il est dit trivial s'il ne contient que $[G]_{\mathbb{D}}$.

Par exemple, lorsque $G$ est monogène, engendré par $h \in \mathbb{D}$ :

$G^{\text {Top }}$ est trivial si $h$ est linéarisable et $\left|h^{\prime}(0)\right|=1$

$G^{\text {Top }}=\mathbb{C} \times G^{\text {For }}$ si $h$ est tangent à $1_{\mathbb{C}}$.

Lorsque $h$ n'est pas linéarisable, on se ramène aisément à ce dernier cas si $h^{\prime}(0)$ est une rotation périodique. Par contre, lorsque $h^{\prime}(0)$ est une 
rotation irrationnelle "proche" des rotations périodiques le calcul de $G^{\text {Top }}$ est un problème ouvert difficile.

Si $G$ n'est pas abélien, $G^{\text {Top }}$ est trivial dès que $J^{1} G$ vérifie des conditions de densité dans $\mathbb{C}^{*}, S^{1}$ ou $\mathbb{R}^{*}[\mathrm{Ce}][\mathrm{Ce}, \mathrm{S}]$.

Ici nous calculons $H^{\text {Top }}$ pour les sous-groupes non abéliens $H$ de $\mathbb{H}$. Le cas abélien ne pose aucune difficulté.

Pour étudier les propriétés des homographies, il est commode d'envoyer $0 \in \mathbb{C}$ sur le point à l'infini, $\infty$, de $\mathbb{C} P(1)$ par le changement de carte $x \rightarrow 1 / x$. Un élément $h \in \mathbb{H}$ s'écrit alors :

$$
h(x)=a x+b, \quad a \in \mathbb{C}^{*}, b \in \mathbb{C} .
$$

Nous identifions $\mathbb{C}^{*}$ au sous-groupe des similitudes $S$ par $a \rightarrow s_{a}$ avec $s_{a}(x)=a x$ et $\mathbb{C}$ au sous-groupe des translations $T$ par $b \rightarrow t_{b}$ avec $t_{b}(x)=x+b$.

Si $H$ est un sous-groupe de $\mathbb{H}$, nous notons $[H]_{H}$ l'ensemble des sousgroupes de $\mathbb{H}$ qui lui sont homographiquement conjugués. Supposons $J^{1} H$ réel et soit $L$ un élément de $\operatorname{GL}(2, \mathbb{R})$. Alors $L_{*} H$ est un sous-groupe de H; c'est l'ensemble des

$$
L_{*} h(x)=a x+L(b) \quad \text { avec } \quad h(x)=a x+b \in H .
$$

ThÉoRÈme 4. - Soit $H$ un sous-groupe non abélien de $\mathbb{H}$. Si

a) $J^{1} H$ n'est pas réel, alors $H^{\text {Top }}$ est trivial;

b) $H=G_{-1,1}=\left\{s_{-1}, t_{1}\right\}$, alors $H^{\text {Top }}=G_{-1,1}^{\text {For }}$;

c) $J^{1} H$ est réel et $H$ n'est pas homographiquement conjugué à $G_{-1,1}$ alors $H^{\mathrm{Top}}=\left\{\left[L_{*} H\right]_{\mathbb{H}} ; L \in \mathrm{GL}^{+}(2, \mathbb{R})\right\}$.

D'après la Proposition 4.1, si $G_{-1,1} \stackrel{\text { Top }}{\sim} G$ avec $G \subset \mathbb{D}$, alors $G$ est formellement conjugué à $G_{-1,1}$. Réciproquement, supposons que $G \subset \mathbb{D}$ soit formellement conjugué à $G_{-1,1}$. Alors $G$ est engendré par l'involution $I(x)=-x$ et un élément $g \in \mathbb{D}_{1}$ formellement conjugué à $x \rightarrow x /(1-x)$ dont le cocycle $c(g)$ est $I$-équivariant. La synthèse de $g$ à partir de $c(g)$ fournit naturellement un homéomorphisme qui conjugue $G$ à $G_{-1,1}$. Ceci prouve le point b) du théorème.

Dans toute la suite de ce chapitre $H$ désigne un sous-groupe non abélien de $\mathbb{H}$ qui n'est pas homographiquement conjugué à $G_{-1,1}$. D'après le Corollaire 4.1 , si $H_{\text {Top }}^{\sim} G$ avec $G \subset \mathbb{D}$, alors $G$ est holomorphiquement conjugué à un sous-groupe $H^{\prime}$ de $H$. Ceci signifie que :

$$
H^{\text {Top }}=\left\{\left[H^{\prime}\right]_{\mathbf{H}} ; H^{\text {Top }} \sim H^{\prime} \text { avec } H^{\prime} \subset \mathbb{H}\right\}
$$

TOME $116-1988-\mathrm{N}^{\circ} 4$ 


\section{Preuve du théorème pour un sous-groupe à deux généra- teurs.}

Proposition 2. - Soit $H_{a}$ le sous-groupe de $\mathbb{H}$ engendré par $t_{1}$ et $s_{a}$ avec $a \neq 1$. Si $H_{a} \stackrel{\text { Top }}{\sim} H$ avec $H \subset \mathbb{H}$ alors $H$ est homographiquement conjugué à $H_{a}$.

Démonstration de la Proposition 2. - Modulo une conjugaison dans $\mathbb{H}$, nous pouvons supposer que $H=H_{a^{\prime}}$ et que l'homéomorphisme de conjugaison $\Phi$ échange $s_{a}$ et $s_{a^{\prime}}$ et laisse fixe $t_{1}$. Il s'agit donc de montrer que $a=a^{\prime}$.

Lorsque $|a|=1$, il est clair que $\left|a^{\prime}\right|=1$. Puisque le nombre de rotation est un invariant topologique, $a$ est alors égal à $a^{\prime}$. Supposons dans la suite $|a|>1$; évidemment on a aussi $\left|a^{\prime}\right|>1$. Montrons que :

(i) Les arguments $\alpha, \alpha^{\prime}$ de a, a sont égaux. $\Phi_{*}$ échange $s_{a *}^{p} t_{1}$ et $s_{a^{\prime} *}^{p} t_{1}$ pour $p \in N$. Ainsi pour $|x|$ assez grand, on a :

$$
\Phi\left(x+a^{p}\right)=\Phi(x)+a^{\prime p} .
$$

En particulier, $\Phi$ échange leurs rayons invariants asymptotiques (voir 0.3.2). $\mathbb{R}^{+} e^{2 i \pi p \alpha}, \mathbb{R}^{+} e^{2 i \pi p \alpha^{\prime}}$ et définit ainsi une application

$$
\varphi: \Delta=\left\{e^{2 i \pi p \alpha} ; p \in N\right\} \longrightarrow \Delta^{\prime}=\left\{e^{2 i \pi p \alpha^{\prime}} ; p \in N\right\}
$$

qui est évidemment bijective. Plus précisément $\varphi$ est monotone en ce sens : si $m_{1}, m_{2}, m_{3}$ sont trois points de $\Delta$ tels que pour une orientation de $S^{1}$ on ait $m_{1}<m_{2}<m_{3}$, pour la même orientation on vérifie que $\varphi\left(m_{1}\right)<\varphi\left(m_{2}\right)<\varphi\left(m_{3}\right)$. Si $\alpha \in \mathbb{Q}$, ceci prouve que $\alpha=\alpha^{\prime}$. Lorsque $\alpha \notin \mathbb{Q}, \Delta, \Delta^{\prime}$ sont denses dans $S^{1}$ et $\varphi$ se prolonge en un homéomorphisme de $S^{1}$ qui conserve l'orientation et conjugue les rotations d'angle $\alpha$ et $\alpha^{\prime}$; par suite, $\alpha=\alpha^{\prime}$.

(ii) $|a|$ et $\left|a^{\prime}\right|$ sont égaux. Soit $C=\{z \in \mathbb{C} ; \rho \leq|z|<|a| \rho\}$ une couronne fondamentale pour $s_{a}$. Pour $\rho$ assez grand, son image $\Phi(C)$ est un domaine fondamental de $s_{a^{\prime}}$. Pour $\rho^{\prime}>0$ et $p \in N$, fixés, assez grands, $\Phi\left(C_{\rho}\right)$ est contenu dans la couronne

$$
C^{\prime}=\left\{z \in \mathbb{C} ; \rho^{\prime} \leq|z|<\left|a^{\prime}\right|^{p} \rho^{\prime}\right\} .
$$

Puisque $\Phi_{*}$ laisse fixe $t_{1}^{n}$, pour $x \in \mathbb{R}^{+}$fixé, assez grand on a :

$$
\Phi(x+n)=\Phi(x)+n \quad \text { si } n \in \mathbb{N} .
$$


D'autre part, il existe $k: \mathbb{N} \rightarrow \mathbb{N}$ tel que $x+n$ appartient à $a^{k(n)} C$ et son image par $\Phi, \Phi(x)+n$, appartient à $a^{\prime k(n)} \Phi(C) \subset C^{\prime}$. Ceci se traduit par les doubles inégalités :

$$
|a|^{k(n)} \rho \leq a+n<|a|^{k(n)+1} \rho
$$

et

$$
\left|a^{\prime}\right|^{k(n)} \rho^{\prime} \leq|\Phi(x)+n|<\left|a^{\prime}\right|^{k(n)+p} \rho^{\prime} .
$$

On en déduit :

$$
\log |a|=\lim _{n \rightarrow \infty} \frac{\log n}{k(n)}=\lim _{n \rightarrow \infty} \frac{\log n}{k(n)+p}=\log \left|a^{\prime}\right| .
$$

4. Preuve du théorème lorsque $J^{1} H$ n'est pas réel. - Soient $H, H^{\prime}$ deux sous-groupes de $\mathbb{H}$ topologiquement conjugués par $\Phi$. Modulo des conjugaisons homographiques et compte-tenu de la proposition précédente, on peut supposer que $t_{1}$ et $s_{a}$ appartiennent simultanément à $H$ et $H^{\prime}$ et que :

$$
\Phi_{*} t_{1}=t_{1}, \quad \Phi_{*} s_{a}=s_{a} .
$$

Nous allons montrer que si $g$ appartient à $H, g^{\prime}=\Phi_{*} g$ est égal à $g$. Notons :

$$
g: x \longrightarrow \alpha x+\beta \quad g^{\prime}: x \longrightarrow \alpha^{\prime} x+\beta^{\prime} .
$$

Les nombres $\alpha, \alpha^{\prime}$ sont invariants par conjugaison homographique. En appliquant la Proposition 2 aux couples $\left(t_{1}, g\right),\left(t_{1}, g^{\prime}\right)$ on obtient déjà $\alpha=\alpha^{\prime}$.

L'homéomorphisme $\Phi$ échange les triplets $\left(t_{1}, t_{a}, g_{1}\right)$ et $\left(t_{1}, t_{a}, g_{1}^{\prime}\right)$, avec

$$
\begin{aligned}
& g_{1}=\left[t_{a}, g\right]: x \longrightarrow x+\rho \beta, \quad t_{a}=s_{a^{*}} t_{1} \\
& g_{1}^{\prime}\left[t_{a}, g^{\prime}\right]: x \longrightarrow x+\rho \beta^{\prime} \quad \text { où } \quad \rho=\frac{a-1}{\alpha} .
\end{aligned}
$$

On peut toujours supposer que $\operatorname{Rel} a, \operatorname{Rel} \rho \beta^{\prime} \geq 0$. Pour $x>0$ fixé, assez grand et pour tout $n \in \mathbb{N}$ on a :

$$
\Phi\left(t_{1}^{n}(x)\right)=t_{1}^{n}(\Phi(x)), \quad \Phi\left(t_{a}^{n}(x)\right)=t_{a}^{n}(\Phi(x)), \quad \Phi\left(g_{1}^{n}(x)\right)=g_{1}^{\prime n}(\Phi(x)) .
$$

Le parallélogramme $P$ de sommets $x, x+1, x+a$ est un domaine fondamental pour le groupe engendré par $t_{1}, t_{a}$. Il existe deux suites $k(n)$, $k^{\prime}(n)$ telles que pour tout $n \in N$ :

$$
g_{1}^{n}(x) \in t_{a}^{k^{\prime}(n)}(P) \quad \text { et donc } \quad g_{1}^{\prime n}(x) \in t_{1}^{k(n)} \cdot t_{a}^{k^{\prime}(n)}(\Phi(P)) .
$$

TOME $116-1988-\mathrm{N}^{\mathrm{O}} 4$ 
Par suite il existe $K, K^{\prime}>0$ tels que pour tout $n \in \mathbb{N}$ :

$$
\left|n \rho \beta-\left(k(n)+k^{\prime}(n) a\right)\right|<K \quad \text { et }\left|n \rho \beta^{\prime}-k(n)+k^{\prime}(n) a\right|<K^{\prime} .
$$

Il en résulte que :

$$
\lim _{n \rightarrow \infty} \frac{k(n)+k^{\prime}(n) a}{n}=\beta=\beta^{\prime}
$$

5. Démonstration du théorème lorsque $J^{1} H \subset \mathbb{R}$. - Soit $H$ un sous-groupe non abélien de $H$ qui n'est pas conjugué à $G_{-1 ; 1}$ et tel que $J^{1} H$ soit réel.

L'ensemble $T_{H}=\left\{b ; t_{b} \in H\right\}$ est un sous-groupe abélien de $\mathbb{C}$ invariant par la multiplication par les éléments de $J^{1} H$. Notant $\bar{T}_{H}$ l'adhérence de $T_{H}$, on est dans l'une des quatre situations suivantes :

$$
\begin{gathered}
\bar{T}_{H}=\mathbb{C}, \bar{T}_{H}=b_{1} \mathbb{R}, \bar{T}_{H}=b_{1} \mathbb{R}+b_{2} \mathbb{Z} \quad \text { et } J^{1} H=\{-1,1\}, \\
T_{H}=b_{1} Z+b_{2} Z \text { et } J^{1} H=\{-1,1\}
\end{gathered}
$$

où $b_{1}, b_{2} \in \mathbb{C}^{*}$ sont $\mathbb{R}$-indépendants. Modulo une conjugaison par un élément de $\mathbb{H}$, nous pouvons supposer que $H$ contient :

la translation $t_{1}$ et que $b_{1}=1$ dans les trois derniers cas, une similitude $s_{a_{0}}$, avec $a_{0} \neq 1$.

Soit $H^{\prime}$ un sous-groupe de $\mathbb{H}$ topologiquement conjugué à $H$ par un homéomorphisme $\Phi$ (défini au voisinage de $\infty$ ). D'après la Proposition 2, nous pouvons supposer que $t_{1}$ et $s_{a_{0}}$ sont invariants par $\Phi_{*}$. D'autre part, $\Phi_{*}$ induit un isomorphisme (continu) $\varphi$ entre les groupes abéliens $T_{H}, T_{H^{\prime}}$ tel que pour $|x|$ assez grand :

$$
\Phi(x+b)=\Phi(x)+\varphi(b) \quad \text { si } \quad b \in T_{H} .
$$

Démonstration de $H^{\prime}=L_{*} H$ avec $L \in \mathrm{GL}^{+}(2, \mathbb{R})$. - Etudions séparément les différents cas :

c $\left.{ }_{1}\right) \bar{T}_{H}=\mathbb{C} ; \varphi$ se prolonge par continuité en $L \in \mathrm{GL}(2, \mathbb{R})$. D'après (*) $\Phi$ est une application $\mathbb{R}$-affine de partie linéaire $L$. Puisque $s_{a_{0}}$ est invariant par $\Phi_{*}$, on a :

$$
\Phi\left(a_{0} x\right)=a_{0} \Phi(x)
$$

et ainsi $\Phi \equiv L$ et $H^{\prime}=L_{*} H$. 
c $\bar{T}_{H}=\mathbb{R} ; H$ est un sous-groupe du groupe $\mathbb{H}_{\mathbb{R}}$ des homographies à coefficients réels; en effet si

$$
g(x)=a x+b \in H,\left[g, s_{a_{0}}\right](x)=x+\left(\frac{1}{a_{0}}-1\right) b
$$

et $b$ est réel. Les mêmes arguments que dans le cas précédent montrent que la restriction de $\Phi$ à $\mathbb{R}$ est un élément de $\operatorname{GL}(1, \mathbb{R})$; c'est en fait $1_{\mathbb{R}}$ puisque la translation $t_{1}$ est laissée fixe. Par suite $H=H^{\prime}$.

c $\left.{ }_{3}\right) T_{H}=\mathbb{Z}+b_{2} \mathbb{Z}$ et $J^{1} H=\{-1,1\}$. Par hypothèse, $H$ contient $t_{1}, s_{-1}$, $t_{b_{2}}$ avec $b_{2} \notin \mathbb{R}$. Si $g \in H$ n'est pas une translation, $s_{-1} \circ g$ en est une. Ainsi $H$ est engendré par $t_{1}, s_{-1}, t_{b_{2}}$ et $\Phi_{*} H=H^{\prime}$ l'est par $t_{1}, s_{-1}, t_{\varphi\left(b_{2}\right)}$.

Montrons que $\varphi\left(b_{2}\right)$ n'est pas réel; $T_{H}$ n'étant pas monogène $T_{H^{\prime}}$ n'est pas monogène. Si $\varphi\left(b_{2}\right)$ était réel $T_{H^{\prime}}$ serait un sous-groupe dense de $\mathbb{R}$ et l'orbite d'un point par $H^{\prime}$ possèderait des points d'accumulation; ce qui n'est pas le cas pour l'orbite d'un point par $H$. Ainsi, $H^{\prime}$ est engendré par $t_{1}, s_{-1}, t_{\varphi\left(b_{2}\right)}$ avec $\varphi\left(b_{2}\right) \notin \mathbb{R}$ et on a :

$$
H^{\prime}=L_{*} H, \quad \text { avec } \quad L \in \mathrm{GL}^{+}(2, \mathbb{R}), L(1)=1, L\left(\varphi\left(b_{2}\right)\right)=\varphi(b) .
$$

c $\bar{T}_{H}=\mathbb{R}+b_{2} Z$ et $J^{1} H=\{-1,1\}$. La preuve précédente montre déjà que $\Phi_{*}$ applique $\left(t_{1}, s_{-1}, t_{b_{2}}\right)$ sur $\left(t_{1}, s_{-1}, t_{\varphi\left(b_{2}\right)}\right)$ avec $\varphi\left(b_{2}\right) \notin \mathbb{R}$. Soit encore $L$ l'élément de $\operatorname{GL}(2, \mathbb{R})$ correspondant $\left(L(1)=1, L\left(b_{2}\right)=\varphi\left(b_{2}\right)\right)$. Comme dans le cas 2, la restriction de $\Phi$ à $\mathbb{R}$ est l'identité et ainsi $H^{\prime}=L_{*} H$.

Nous avons donc établi dans le cas où $j^{1} H$ est réel que la conjugaison topologique implique la conjugaison via un élément de $\mathrm{GL}(2, \mathbb{R}$ ) (à distance finie via des homéomorphismes de type $z \rightarrow L_{\infty}(z)=1 / L(1 / z)$, $L \in \mathrm{GL}(2, \mathbb{R}))$. On a ainsi prouvé que :

$$
H^{\text {Top }}=\left\{\left[L_{*} H\right]_{\mathbf{H}} ; L \in \mathrm{GL}^{+}(2, \mathbb{R})\right\}
$$

On remarque que $H^{\text {Top }}$ s'identifie à un quotient de l'espace $\mathrm{M}$ des modules du Tore $\mathbb{T}^{2}$. Dans le cas $c_{2}$ ) où $\bar{T}_{H}=\mathbb{R}, H^{\text {Top }}$ est trivial, dans le cas $c_{3}$ ), $H^{\text {Top }}$ est précisément $\mathbb{M}$ (position du réseau $T_{H}=Z+b_{2} Z$ ). Les cas $c_{1}$ et $\mathrm{c}_{4}$ sont intermédiaires.

Remarques. - Les résultats topologiques obtenus ici s'appliquent aussi aux diagrammes et aux équations différentielles. Donnons deux exemples de telles applications :

1) un diagramme $d=\left(f_{1}, f_{2}\right)$ topologiquement conjugué au diagramme $\left(x^{2}, x^{3} /(1+x)^{3}\right)$ lui est holomorphiquement conjugué; en fait on

$$
\text { TOME } 116-1988-\mathrm{N}^{\circ} 4
$$


peut classifier les diagrammes ensemblistement conjugués à $\left(x^{2}, x^{3} /(1+\right.$ $x)^{3}$ ).

2) une équation de Pfaff $\Omega=a d x+b d y$ topologiquement conjuguée à $\Omega_{R}=d\left(y^{2}+x^{3}\right)+x(2 x d y-3 y d x)$ lui est holomorphiquement conjuguée; ceci résulte du fait que $\Omega_{R}$ conduit après résolution à une équation de Ricatti, son holonomie projective étant de type $(-x, j x /(1+x))$.

Note. - E. Ghys nous a signalé, après la remise de notre manuscrit, un article de A.A. Shcherbakov, topological and analytical conjugacy of non commutativ groups of germs of conformal mappings. Trudy Seminera Imeni, I, G, Pertrovskogo, $\mathrm{n}^{\circ} 10$, p. 170-196, (1984), dans lequel il est montré le théorème suivant :

ThÉoRÈme. - Soient $G, G^{\prime}$ deux sous-groupes de $\mathbb{D}$ topologiquement conjugués. Alors si $G$ est non résoluble, $G$ et $G^{\prime}$ sont holomorphiquement conjugués.

D'autre part, on vérifie aisément qu'un sous-groupe résoluble $G$ non commutatif de $\mathbb{D}$ est formellement conjugué à un sous-groupe dont les éléments s'écrivent $a \exp t X_{p, 0}$ où $p \in \mathbb{N}$ est fixé et où $a, t \in \mathbb{C}$. C'est-àdire que $G$ est formellement conjugué ou relevé par $z \rightarrow z^{p}$ d'un sousgroupe du groupe $\mathbb{H}$ des homographies. Il est clair que nos résultats sur la classification de ces sous-groupes se "relèvent" par $z \rightarrow z^{p}$.

Ainsi nos résultats complètent agréablement le théorème de A.A. Shcherbakov et permettent d'obtenir une description complète de l'espace des modules (topologique-formel) des sous-groupes non abéliens de $\mathbb{D}$.

\section{BIBLIOGRAPHIE}

[C] CAMACHO (C.). - On the local structure of conformal mappings and holomorphic vector fields in $\mathbb{C}^{2}$, Astérisque, t. 59-60, 1978, p. 83-94.

[Ce] Cerveau (D.). - Espaces de modules de certaines équations différentielles du premier ordre. preprint Dijon, 1986.

[Ce,S] Cerveau (D.) et SAD (P.). - Problèmes de modules pour les formes différentielles singulières dans le plan complexe, Comment. Math. Helv., t. 61, 1986, p. 222-253.

[D] Dufour (J.-P.). - Diagrammes d'applications différentiables. Thèse Montpellier, 1979 .

[Du] DulAC (H.). - Recherches sur les points singuliers des équations différentielles, J. École Pol., t. 2,9, 1904, p. 1-125.

[E $\left.\mathrm{E}_{1}\right]$ ECALle (J.). - Les fonctions résurgentes. Tome II Les fonctions résurgentes appliquées à l'itération. - Publ. Math. d'Orsay, 1981. 
$\left[\mathrm{E}_{2}\right]$ Ecalle $(\mathrm{J}$.$) . - Les fonctions résurgentes. Tome III L'équation du pont et la$ classification analytique des objets locaux. - Publ. Math. d'Orsay, 1985.

[H] Herman (M.). - Recent results and some open questions on Siegel's linearisation theorem ..., preprint Centre Math. École Polytechnique, 1986.

[L] LiNs (N.). - Construction of singularities of holomorphic vector-fields in $\mathbb{C}^{2}$ with a given holonomy. - Preprint IMPA.

[Ma] Malgrange (B.). - Travaux d'Ecalle et de Martinet-Ramis sur les systèmes dynamiques, Séminaire Bourbaki, [Exposé n ${ }^{\circ} 582$ ], novembre 1981.

$\left[\mathrm{M}, \mathrm{R}_{1}\right]$ MARTINeT (J.) et RAmis (J.-P.). - Problèmes de modules pour des équations différentielles non linéaires du premier ordre, Publ. Math. IHES, t. 55, 1982, p. $63-124$.

$\left[\mathrm{M}, \mathrm{R}_{2}\right]$ MARTinet (J.) et RAmis (J.-P.). - Classification analytique des équations différentielles non linéaires résonnantes du premier ordre, Ann. Sci. École Norm. Sup., t. 16, 1983 , p. 571-621.

[M,M] Mattei (J.-F.) et Moussu (R.). - Holonomie et intégrales premières, Ann. Sci. École Norm. Sup., t. 13, 1980, p. 409-523.

[Mo] Moussu (R.). - Holonomie évanescente des équations différentielles dégénérées transverses. In singularities and Dynamical systems. - North Holland 1985, pp. 151-173.

[Mo] Moussu (R.). - Lettre de Moussu à Martinet.

[P] Poincare (H.). - Mémoires sur les courbes définies par une équation différentielle, J. Math. Pures Appl., t. 3.7, 1881, p. 375-442.

[R] RAMIS (J.-P.). - Preprint IMPA, 1986.

[S] SiEgEL (C.L.). - Über der normal Form analytischen Differentialgleichungen in der Näher einer Gleichgewichtslösung. Göttingen Nachrichten des Wissenschaften, 1952, pp. 21-30.

[T] Takens (F.). - Singularities of vector fields, Publ. Math. IHES, t. 43, 1974, p. $47-100$.

[V] Voronin (S.M.). - Analytic classification of pair of involutions and its applications, Functional Anal., t. 16, $\mathrm{n}^{\circ} 2$, 1982, p. 94-100. 